(Aus dem I. anatomischen Institut in Berlin.)

\title{
Ueber die Entwicklung und morphologische Bedeutung der „Pseudobranchie“ und ihrer Umgebung bei Lepidosteus osseus.
}

\author{
Von
}

\author{
Friedrich W. Miiller, \\ Dr. med. in Berlin. \\ Hierzu Tafel XIX und XX.
}

\section{Einleitung.}

Es ist bekannt, dass sich am Kiemendeckel von Lepidosteus zwei Kiemen finden, welche einen gleichartigen Ban besitzen und mit ihren Enden an einander stossen. À diese Kiemen kniipft sich eine literarische Discussion, indem von der einen Seite behauptet wird, dass es sich dabei um zwei verschiedene Kiemen (Hyoid- nnd Spritzlochkieme) handle, während von der anderen Seite die Meinung vertreten wird, dass es zwei Stuicke e i n e r Kieme, der Hyoidkieme, seien. Auf der einen Seite stehen Joh. Müller, Dohrn u. A., anf der anderen Seite Ge genba ur, Balfour, Wright. Zur Aufklärung der Frage hat man nicht allein die Kiemen selber verwerthet, sondern auch die zt- und ausfuihrenden Gefüsse derselben und die Verbindung der letzteren mit den Kopforefässen, die Spritzlochtasche und die Nerven heranzmziehen versucht; es fehlt jedoch der Nachweis der Genese der Kiemen selbst. Schon von anderer Seite (B a l f o u r und Parker, Wright) ist hervorgehoben worden, dass diese Kiemen verhältnissmüissig spüt, man kann sagen, auffallend spät hervortreten, worauf im Wesentlichen der negative Ausgang. der früheren Untersnchungen zurïckzuführen ist. Ich befand mich jedoch in der gliicklichen Lage, durch Benutzung eines von den Herren H. Virchow und $\mathbf{F}$ ülleborn gesammelten Materials die geeigneten Stadien für die Lösung dieser Aufgabe zu finden.

Ueber die Literatur dieses Gegenstandes und die Erörterung der morphologischen Fragen ist von $A$. Dobrn in seiner 


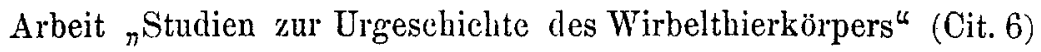
mit Abdruck der Originalstellen der einschlägigen Abhandlungen so ausführlich geschrieben, dass eine Recapitulation derselben wohl eine lästige Wiederholung wäre; ich gehe deswegen hier nicht genauer auf die Literatur ein, sondern werde die Arbeiten im Verlaufe der Untersuchungen citiren.

Bevor ich nun meine Ergebnisse darstelle, will ich zunächst die nöthigen Nachweise über Material und Technik geben und im Interesse der Klarheit die von mir gebrauchten Ausdrücke definiren.

Material und Technik: Das Material, welches mir für diese Untersuchungen in liebenswürdigster Weise von Herrn Prof. Hans Virchow zur Verfügung gestellt wurde, ist theils von dem genannten Herrn selbst am Blake Lake im Staate NewYork, theils von Herrn Fül le bor n in Oconomowoc (Wisconsin) gesammelt worden (Cit. 10). Es ist in beiden Fällen dieselbe Species, Lepidosteus osseus, und die Exemplare zeigen in den entsprechenden Stadien nur den Unterschied, dass die des $F \mathfrak{u} 11 \mathrm{e}$ b or n'schen Materials infolge künstlicher Fütterung etwas kleiner sind als die anderen. Zur Conservirung wurden die verschiedensten Reagentien angewendet: Chromsäure, Müll er'sche Flüssigkeit, Sublimat, Formalin und Platinchlorid. Die Form zeigte sich am besten erhalten bei den in Chromsäure geliarteten Objekten, und ich wählte deswegen, da es mir hauptsächlich auf die Form ankommen musste, diese. Für die vorliegende Arbeit wurden die Stadien I-V aus dem Material des Herm Vir chow, als VI. ein Exemplar von dem übrigen Material gewählt. Bei der Verarbeitung zeigte sich, dass die Gestalt der Organe, die Form der Zellen u. s. w. vorzüglich erlualten, dass aber die Fürbbarkeit des Materials sehr beeinträchtigt war, wahrscheinlich durch das lange Liegen; es färbte sich im Schnitt mit keiner der üblichen Kernfarben, sondern es musste die $\mathrm{Heid}$ enhain'sche EisenHämatoxylinmethode angewendet werden, welche zwar ziemlich viel Zeit in Anspruch nahm, aber auch sehr gute Resultate ergab (vergi. die Querschnittzeichnungen).

Die Verarbeitung der einzelnen Stadien geschah in der Weise, dass zunächst genane Zeichnungen des Thieres im auffallenden und im durchfallenden Lichte bei bestimmter Vergrösserung angefertig't wurden. Das Schneiden der Objekte ge- 
Ueber die Entwicklung und morphologische Bedeutung etc. 465

schah bei Paraffineinbettung nach Aulegung von Richtebenen und -linien zum Zweck der Reconstruktion. Als Schnittdicke nahm ich zuerst $15 \mu$ an, um ein Zusammenschieben der Schnitte zu verhindern, und kam damit vollständig bei den älteren Stadien (V und VI) ans. Für die jüngeren Stadien war aber auf $15 \mu$ Schnitten wenig zu sehen and deswegen wurde diese in Serien zu $10 \mu$ geschnitten. Um eine genaue Orientirung über die Topographie der Organe zu erhalten, wurden mebrere Stadien in drei Ebenen geschnitten: sagittal, frontal und quer, die anderen wurden quer zerlegt, wie auch die Reconstruktionen nur nach Querschnitten angefertigt wurden. $\mathrm{Da}$ es aus den Schnitten allein, zumal bei einem fast unbekannten embryologischen Material, kaum möglich war, eine genaue Vorstellung, von dem Verlauf und der Lage des Spritzlochganges zu bekommen, wurden zwei Stadien plastisch reconstruirt, von denen aber nur das ältere (Fig. 14) eine Spritzlochtasche resp. -Gang ergab, während das jüngere kaum irgend welche Besonderheiten zeigte. Die Reconstruktion erfolgte nach der Born'schen Wachsplattenmethode, und zwar wurde jeder einzelne Schnitt gezeichnet und als Wachsplatte ausgearbeitet.

Die Gefässbilder sind auf die Art gewonnen, dass sämmtliche Schnitte der Serie mit allen Gefässdurchschnitten gezeichnet wurden und dann durch Abmessungen der Entfernungen von der Medianebene resp. der Richtebene der Verlanf der Gefässe eruirt wurde. Auf photographischem Wege wurden durch Verkleinerung sodann diese Figuren auf die bestimmte Grösse gebracht. Die Organe des Kopfes sind nur in den Umrissen gegeben um die Vorstellung von der Lage der Gefässe zu erleichtern. Zugleich habe ich die Zeichnungen soweit ausgeführt, dass in den wichtigsten Stadien die Form n. s. w. des Kopfes deutlich wird und diese Zeichnungen bis zu einem gewissen Grade das Oberflächenbild ersetzen. Die Schnittzeichnungen (Figg. 1, 4, 7, 8, 9) sind mit der Feder auf das Genaneste unter dem Mikroskop gezeichnet und zur Controle photographirt.

\section{Ausdrücke und Bezeichnungen.}

Ich unterscheide:

Spritzlochgang, -strang, -tasche.

Visceralbogen - Kiemenbogen. 
Bogengefässe - Kiemengefässe.

Zuführende - ausfïhrende Kiemenarterien.

Dorsales und ventrales Randgefäss des Kiemendeckels.

A. branchio-opercularis nenne ich die Fortsetzung der ventralen Verlängerung der ersten ausführenden Kiemenarterie innerhalb der lateralen Rachenwand bis zur Vereinigung mit der A. hyoideo-opercularis. Das vereinigte Stück bis zur Einmündung. in das ursprüngliche erste Visceralbogengefäss bezr. bis zur Stelle der späteren Pseudobranchie: A. afferens spiracularis.

Als A. efferens spiracularis bezeichne ich die Carotis interma Joh. Müller's.

\section{Untersuchungsergebnisse.}

\section{Stadium.}

O b e r flä ch e n bild: Die jüngste Larve, welche zu diesen Untersuchungen verwendet wurde, ist sogleich nach dem Ausschlüpfen conservirt. Sie hat eine Länge von $8 \mathrm{~mm}$ und besitzt einen Dottersack, der noch etwa die Hälfte des Thicres einnimmt $(3,8 \mathrm{~mm})$. Die Körperform ist plump und erinnert anffallend an die eben ansgeschlïpfte Froschlarve (Cit. 10). Im Leben ist diese Aehnlichkeit noch grösser, da beide Larvenarten mittels einer Saugscheibe sich an der Unterseite von Pflanzen, Steinen u. s. w. festhalten. Der Kopf ist kurz und wenig gegliedert, seine Organe treten nur schwach hervor. Hinter der am Vorderende sitzenden Sangscheibe liegt die annühernd viereckige Mundüffnung ganz dicht vor dem Dotterorgan; Unterkiefer sind noch nicht angedeutet. Die erste Kiemenspalte ist bereits vollständig geöffnet und dadurch der Kiemendeckel, welcher vor derselben liegt, schärfer abgesetzt. Er hat, wie sich bei der Betrachtung von oben zeigt, die Form eines sehr stumpfwinkligen Dreiecks, dessen Basis von ventral und vorn dorsal- und rückwärts verlïnft; in Seitenansicht, wovon Fig. 2 einen ungefähren Eindruck giebt, erscheint er in Verkürzung als ein S-förmiger Wulst. Die Kiemenbogen werden ganz von ihm bedeckt, sonst wiirde man vielleicht schon den ersten und wweiten Bogen getrennt finden, da sich zwischen ihnen bereits eine Furche, wenn auch noch keine Spalte zeigt. Zwischen dem Kiemendeckel und dem Auge ist die Oberfläche rollkommen glatt und zeigt keinerlei Oeffunng, die dem 
Ueber die Entwicklung und morphologische Bedeutung etc. 467

Spritzloch entsprechen köunte, nicht einmal eine Einsenkung: Das hintere Körperende verläuft in gerader Richtung und ist hinter dem Dotterorgan dorsal und ventral von einem Flossensaum umgeben, der nach hinten zu breiter wird, aber das Ende der Chorda nur wenig überragt; die späteren unpaaren Flossen sind durch Pigmentirung angedeutet. Die Analöffnung liegt etwa in der Mitte zwischen Dottersack und Schwanzspitze.

Ergebniss der Serie: 1. Anlage des Spritzlochkanales: Mitten zwischen Hinterrand des Anges und Vorderende des Gehörorgans sieht man in dem Winkel, welchen die laterale und dorsale Wand der Rachenhöble nit einander bilden, eine ganz flache Einsenkung, von welcher ein blinder Strang lateral- und dorsalwärts und zugleich, wie die Verfolgung der Serie lehrt, ein wenig rückwärts geht. Dieser Strang ist auf sechs Schritten $(10 \mu)$ zu verfolgen und zwar so, dass die ersten zwei das pharyngeale Stiuck, die letzten beiden das laterale enthalten, während auf den mittleren die Zellenreiben, welche den Strang bilden, vollständig getroffen sind. Daraus geht hervor, dass der Strang die Gestalt eines Cylinders hat, welcher von vorn medial und ventral lateral-, dorsal- und rückwärts zieht. Er erreicht die Epidermis, ohne mit ihr in Verbindung zu treten, letztere zieht vielmehr ohne Veränderung ihres Charakters und ohne Aenderung des Niveaus ïber denselben hinweg (Fig. 1). Ein Lumen fehlt dem Strange gänzlich; derselbe ist gebildet von zwei Reihen Zellen, welche eine Fortsetzung der tieferen Zellenlage des zweischichtigen Rachenepithels sind, nur an dem kegelförmigen, pharyngealen Ende des Stranges findet sich auch eine Einstülpung der oberflächlichen Lage des Pharynxepithels in Gestalt eines Zapfens (s. Fig. 1). Zu diesem Strange tritt von dorsal und hinten ein Nerv, welcher sich nur bis zu den Zellen desselben, nicht weiter verfolgen lässt, und dessen Beziehtung zu dem Strange durch die späteren Stadien klar wird (Fig. 1 R. o.).

2. Ki eme $n$ b og e $n$ : Die erste Kiemenspalte, d. h. die Spalte zwischen dem Hyoidbogen und dem ersten Kiemenbogen ist, wie elwähnt, vollkommen durchgängig; die Trennung zwischen dem ersten und zweiten Kiemenbogen ist, wie gleichfalls erwähnt, durch eine Furche angedentet, aber nicht durchgeführt. Von 
dem dritten und vierten Kiemenbogen ist noch nichts $z u$ sehen, ebensowenig findet sich eine Spur von Kiemenblättchen.

3. Herz und Gefässe. a) $\mathrm{Herz}$ : Das Herz hat eine Lage, welche von der bei älteren Stadien abweicht und durch die Grösse und Gestalt des Dotterorgans bedingt ist. Die Längsachse steht fast senkrecht, ist aber eher etwas dorsal- und riickwärts geneigt, sodass der Winkel zwischen dem Truncus und den Kiemenbogen älterer Stadien ausgeglichen ist (Fig. 2 Co.).

b) Truncus: Der Truncus ist ein sehr kures, dickes Gefäss, da sich die ans ihm hervorgehenden Aeste sofort hinter dem Herzbentel abzweigen (Fig. 3 Tr.).

c) Bogengefässc: Es griebt vier bogenförmige Gefässe in 'diesem Stadium, welche von der ventralen Seite ans dem Truncus nach der dorsalen Seite emporbiegen, um hier die Aorta und Carotis zu bilden. Aus dem Truncus entspringen sic mit zwei Stämmehen, von denen das erste (arc. $I+I I$, Fig. 3) die beiden vorderen, das zweite (arc. $I I I+I V$, Fig. 2 u. 3) dic beiden hinteren Bogengefäisse entsendet. Das erste verläuft seitwärts ${ }^{1}$ ) (s. Fig. 3 arc. $I+I I$ ) und theilt sich vor der nuteren Ecke der Kiemendeckelbasis in die beiden crsten Bogengefässe (Fig. 2 u. Fig. 3 arc. $I$ u. arc. $I I)$. Von diesen biegt das vordere (arc. $I$ ) in derselben Querebene empor und erreicht vor der Anlage des Spritzlochganges die Carotis (Ca.).

Das zweitc Bogengefüss verlänft rückwärts nud aufwärts an Rande des Kiemendeckels und tritt hinter der Anlage des Spritzlochkanales in die Carotis ein. An diesem Bogengefässe (arc. $I I$ ) findet sich eine Anastomose (an.) znm vorderen Bogen und nach hinten hin eine im Kiemendeckel gelegene Schleife, von welcher noch zwei kurze Aeste ausgehen, die in der oberen Ecke des Kiemendeckels endigen (Fig. 2 n. 3 arc. $I I$, arc. $I I^{\prime}$ ).

Das hintere Stämmchen (arc. $I I I+I V$ ) ist kürzer als das vordere und breiter, ansserdem rückwärts gerichtet; es spaltet sich in zwei gleich starke Aeste (arc. III u. arc. IV). Diese biegen der Medianebene näher, als das eben beschriebene Bogengefäss nach der dorsalen Seite um und treten, rückwärts gerichtet, ohne seitliche Gefässe abgegeben zu haben, in übereinstimmender Weise an die Aorta (Ao.) heran.

1) Auf Fig. 2 ist es nicht sichtbar, da es von dem aufsteigenden Aste verdeckt wird. 
Ueber die Entwicklung und morphologische Bedeutung etc. 469

d) Carotis und A o r ta: Aus der Vereinigung der vier Bogengefässe geht ein paariger dorsaler Längsstamm hervor, der die Carotis und Aorta enthält. Im Gegensatz zu späteren Stadien ist also bemerkenswerth, dass die beiden Aorten noch bis hinter die Einmtindung der Aa. effer. branch. II (arc. IV) getrennt sind und davon hängt es auch $a b$, dass die Aorta in der geraden Verlängerung der Carotis liegt. Da eine Anastomose zwischen beiden Carotiden fehlt, so ist die Abweichung des Bildes von dem des bekaunten Kopfkreises der Selachier und Teleostier sehr erheblich. Fig. 3 zeigt, dass das dritte und vierte Bogengefäss in die Aorta rückwärts, das erste dagegen nach vorn in die Carotis gerichtet ist, während das zweite ungefähr rechtwinklig auf das hintere Stuick der Carotis auftrifft. Man kann daraus wohl direkt anf die Strömungsverhältnisse schliessen. Das vorderste Stück der Carotis berührt auf seinem Verlaufe.die einzelnen Organe des Kopfes und giebt kurze Aeste an dieselben ab (Fig. 2 u. 3 o.n.).

\section{Stadium.}

Oberflächenbild: Die nächste untersuchte Larve ist zwar nur wenig älter, zeigt aber gegen die vorige ganz erhebliche Unterschiede. Der ganze Körper ist mehr in die Länge gezogen, die Formen sind schlanker. Die Länge misst $10,9 \mathrm{~mm}$, die des Dotterorgans $4,3 \mathrm{~mm}$; letzteres ist schmaler und länger geworden, wenn auch relativ, d. b. im Verhältniss zur Körperlünge, kürzer als im ersten Stadium. In dorsoventraler Richtung ist der Durchmesser gleich geblieben; die Dottermenge hat also nicht ab-, scheint vielmehr zugenommen zu baben, was bei anderen Wirbelthieren Analogieen findet und nur durch Wasseraufnahme zu erklären ist, da ja zugleich ein Theil des Dotters für den Aufbau des Körpers verbraucht wird. Der Kopf ist stark nach vorn gewachsen, seine Organe treten deutlicher hervor. Die Saugscheibe ist noch vollständig erhalten und zeigt die Saugnäpfe; zwischen ihr. und dem Ange liegt die einfache Nasenöffnung und zwar direkt an der vorderen (nasalen) Seite desselben. Das Auge ist gross, die Stelle der Linse ist anch bei conservirten Larven deutlich durch Pigmentmangel innerhalb eines pigmentirten Ringes zu unterscheiden. Die Gehirnabschnitte sind wie beim I. Stadium von aussen gut zu erkennen und werden 
bei der Betrachtung im durchfallenden Lichte noch dentlicher. Hinter der Saugscheibe liegt ventral die ziemlich kleine Mundöffnung, hinter welcher die Anlagen der Unterkiefer vor dem Dotterorgan als rundliche Wülste hervorspringen. Eine ganz rapide Entwicklung hat während dieser Zeit der Kiemendeckel durchgemacht. Seine Basis steht fast senkrecht zur Lüngsachse des Körpers und beginnt dorsal hinter der Wölbung der Gehörkapsel, ventral wwischen Unterkiefer und Vorderende des Dotterorgans. Seine Form $^{1}$ ) ist jetzt die eines rechtwinkligen Dreiecks, die Fläche ist stark nach lateral convex gekrümmt. Dic Kiemenbogen werden bis auf eine kleine Ecke am oberen Rande, wo zwei Kiemenspalten eben sichtbar sind, vollständig bedeckt, sodass sich die Zahl der Kiemenbogen von aussen nicht erkennen lässt. Das Herz ist durch einen Wulst am vorderen Ende des Dotterorgans angedeutet, seine genaue Lage aber ist crst ans den Séhnitten abzuleiten. Die vordere Extremität hat eine gerade Basis und einen halbkreisfürmigen Rand und beginnt vorn in derselben Querebene, in der dic Spitze des Kiementeckels liegt; die beiden Extremitäten divergiren nach hinten zu. Der iibrige Theil des Körpers zeigt dureh Pigmentirung dentlich dic Eintheilung in Muskelsegmente, von denen man, bei dem cinen der untersuchten Exemplare, welches quer gesehnitten wurle, 65 mit Sicherheit erkennen kann. Der Flossensaum hat noch dieselbe Ansdehnung wie beim vorigen Stadium, zeigt aber. schon an den Stellen der späteren unpaaren Flossen Verlureiterungen und Pigmentflecken. Die hinteren Darmabsehnitte zeigen wenig Unterschiede gegen das frühere Verlhalten.

Ergebniss der Serie. 1. Anlage des Spritzlochkanals: Die Anlage des Spritzlochkanales ist in ihrer Entwicklung gegen dic äussere Form und die übrigen Organe stark zuritckgeblieben; die Einsenkung des Rachenepithels ist nur wenig stärker als im I. Stadinm und der Zellenzapfen aus der oberflïehlichen Schicht des Epithels ist etwas weiter lateralwärts gewachsen, wodurch der pharyngeale Theil des Zellstranges trompetenartig erweitert wird; sonst sind die Verhältnisse unverändert (Fig. 4).

1) Auf Figur 5 u. 6 ist das hintere Ende nach innen umgeschlagen, daher die geradlinige Begrenzung. 
Ueber die Entwicklung und norphologische Bedeutung etc. 471

2. Ki e m e n bogen: Sehr erhebliche Veränderungen zeigen sich naturgemäss im Verhalten der Kiemenbogen und der Gefässe. Es sind bereits vier Kiemenbogen angelegt, von denen die drei ersten schon Kiemenblïttchen von fast rundem Querschnitt ohne die feineren, secundären Blättchen besitzen. Der vierte Bogen ist hinten noch nicht durch eine Spalte von der Pharynxwand getrennt, er liegt als kleiner Wulst vor dem Eingang in den Schlund.

3. Herz und Gefäise. a) Herz: Durch die Verkleinerung des Dotterorgans und das Wachsthum des Kopfes nach vorn bat die Lage des Herzens eine wesentliche Veränderung: erfahren. Die, Spit\%e ist nach hinten gerichtet, der Truncus nach vorn, sodass die Achse jetyt in Gegensatz zu dem I. Stadium vorn ventral und hinten dorsal- und vorwärts gerichtet ist. Der Blutstrom erführt dadurch eine Knickung und verläuft in einem stumpfen, nach hinten offenen Winkel (Fig. う Co.).

b) Tru n cus: Der 'Truncus liegt wie beim vorigen Stadium vor der Spitze des Dotterorgans und ist gleichfalls sehr kurz und dick, da sich die Gefässe sogleich verzweigen (Fig. $6 \mathrm{Tr}$.).

c) Bogengef ìsse: Ausserordentlich wichtige Abweichungen zeigt dieses Stadium gegen das jüngere in Bezug auf die Bogengefässe. Wie envähnt, ist die Zahl der wahren Kiemonbogen auf vier vermehrt, die Gesammtzahl der angelegten Bogen ist also sechs. Von diesen sind die beiden vordersten am stärksten verändert; aus dem Truncus $(T r$.) entspringt wie beim I. Stadium ein starker Stamm ( $h . v$.), der ebenso zuerst genau lateralwärts verläuft, sich dann aber nicht in zwei sich dorsalwärts wendende Aeste theilt, sondern mit mehreren Verzweigungen, die sämmtlich nach hinten verlaufen, ein grosses Netz feiner Capillaren ${ }^{1}$ ) auf dem ventralen Theile des Kiemendeckels bildet.

Die beiden früheren dorsalen Endstücke dieses Stammes sind auch jetzt noch crkennbar. Sie erscheinen in diesem Stadium als zwei starke Gefüsse, von denen- das eine (d. $l$.$) vor,$ das andere (d. II.) hinter der Anlage des Spritzlochkanales in die Carotis mündet. Die Verbindung nit dem ursprünglichen gemeinsamen Stamm (h. v.) ist jetzt durch feine Aeste bergestellt,

1) Diese Verzweigungen des ventralen Randgefässes des Kiemendeckels sind in der Zeichnung tortgelassen. 
von denen der eine, läng'ste, dem früheren Hauptgefäss zu entsprechen scheint. Diese feineren von der ventralen Seite herkommenden Gefässe vereinigen sich $\%$ einem in der lateralen Rachenwand liegenden Querast (Fig. 5 n. 6 Qu.), der direkt mit dem ersten und durch eine Anastomose (Fig. 5 u. 6 an.) mit dem zweiten Bogengefäss (-Rest) in Verbindung steht. In den zweiten Bogen mündet ausserdem noch ein stärkerer Ast von ventral und einer vom dorsalen Rande des Kiemendeckels kommender ein (Fig. 5 u. 6 h. d.). Zwischen allen Aesten der oberen Bogengerässtheile und dem ventralen Stammstiicke besteht ein sehr reiches Netz von Anastomosen und Capillaren.

Der Unterschied zwischen den beiden Stadien ist also der, dass die direkte Verbindung zwischen dem ventralen Anfangsstïck und den dorsalen Endästen aufgehört hat und nur noch Capillaren die frühere Zusammengehörigheit andeuten. Die erwähinte Anastomose zwischen dem ersten und zweiten ursprünglichen Bogengefässe ist für die Erklärung der späteren Stadien wichtig.

Nach der Abgabe des Stammes für die beiden ersten Visceralbogen theilt sich der Trunens sogleich in zwei Hauptstänme, die beiderseits symmetrisch verlaufen (Fig. 5 u. 6) und vier Acste abgeben; die ersten drei derselben sind die zufïhrenden Arterien für den ersten bis dritten Kiemenbogen (Fig. 5 u. 6 $a f f . b r . I-I I I)$, sie lösen sich allmählich in dic Kicmencapillaren auf und endigen unterhall des dorsalen Endes der Kiemenbogen. Der vierte Ast geht in S-förmiger Krïmmung durch den wulstförmigen vierten Kiemenbogen, löst sich aber nicht auf, da dieser Bogen noch keine Kiemenblättchen besitzt, sondern geht unverandert hindurch. Die erste Andeutung einer Trennung in zuund ausführende Arterie zeigt sich in einem kleinen blinden Gefässzweige, der vom dorsalen Drittel dieses Bogengefässes ventralwärts läuft. Die drei ersten ausführenden Kiemenarterien (Fig. 5 u. 6 eff. br. I-III) setzen sich aus den Kiemencapillaren zusammen und ergiessen sich in die Aorta (Fig. 5 u. 6 Ao.), welche nur noch vor dem zweiten Kiemenbogen gespalten ist. In die dritte ausführende Kiemenarterie mündet das unverzweigte Gefäss des vierten Bogens.

d) Aorta und Carotis: Die Aorta zeigt gegen das I. Stadium nur den Unterschied, dass die Vereinigung der beiden 
Ueber die Entwicklung und morphologische Bedeutung etc. 473

urspringlichen Aortenwurzeln schon vor dem zweiten Kiemenbogen erfolgt. Die Carotis communicirt mit dem entsprechenden Gefäss der anderen Seite nicht und giebt ebenfalls kurze Aeste an die Organe des Kopfes ab (Fig. 5 u. 6 o. n. ce.).

\section{Stadium.}

Oberflä chenbild: Das III. Stadium zeigt in der Ent. wicklung seiner äusseren Gestalt den Uebergang vom II. zum IV. und hat keine Besonderheiten, welche eine ausführliche Beschreibung nöthig machten. Dagegen findet sich bei den einzelnen Organen mancherlei Veränderung, so dass die Serie von einiger Wichtigkeit ist.

Ergebniss der Serie. 1. Anlage des Spritzlo chganges: Der die Anlage des Spritzlochganges bildende Zellstrạng zeigt sich gegen das vorige Stadium verändert. $\mathrm{Er}$ ist bedeutend länger geworden, erreicht aber mit seinem lateralen Ende nicht mehr die Epidermis, sondern ist am Ende etwas dorsalwärts emporgebogen ${ }^{1}$ ). Das pharyngeale Ende hat sich erweitert, so dass jetzt eine Tasche besteht, die etwa ein Viertel der ganzen Anlage einnimmt. Ein wichtiger Unterschied tritt in diesem Stadium hinsichtlich der Auskleidung der Tasche bzw. der Zelllagen in dem Strange auf. Die laterale, vordere und hintere Wandung ist mit ebendemselben Epithel versehen, wie es die Rachenhöhle besitzt, die mediale Seite des Stranges zeigt dagegen eine Lage hoher cylindrischer Zellen, welche sich viel dunkler färben, als die anderen und deswegen deutlich hervortreten (Fig. 7).

Der Nerv, dessen Bezichung zu der Anlage des Spritzlochkanales erwähnt wurde, ist auch in diesem Stadium gut zu verfolgen. Fig. 7 zeigt auf der rechten Seite den Nerven (R.o.) in der Mitte der Gehörkapsel, durch welche er in einem engen Kanal zur lateralen Seite hindurchtritt; auf der linken Seite ist eine etwas weiter vorn gelegene Stelle getroffen; der Nerv hat bereits die Gehörkapsel verlassen und sein Querschnitt liegt ein wenig dorsalwärts von dem Zellstrange. Er lässt sich auf den folgenden Schnitten erkennen und tritt ganz nahe an die Zellen

1) Ist auf Figur 7 nicht zu sehen, da es in einem der folgenden Schnitte getroffen ist. 
der Anlage heran. Vor dem Zellstrange ist keine Spur mehr von dem Nerven zu sehen.

Auf Kiemenbog'en, Hel's u. s. w. gehe ich nicht näher ein, da nur die Veränderung vorliegt, dass der vierte Kiemenbogen frei ist und schon einige Kiemenblattchen besit\%t.

B ogengef äs se: Viel wichtiger haben sich die Verhälnisse an den beiden ersten Visceralbogen gestaltet. Der dorsale Theil des ersten Bogengefässes hat sich wenig verändert. Die Anastomose zum zweiten Bogengefäss hat sich starker entwickelt und bildet jetzt die eigentliche Fortsetzung des zum zweiten Bogen ziehenden dorsalen Randgefässes des Kiemendeckels; das ursprüngliche zweite Bogengefäss ist ganz schwach geworden. Einen äusserst wichtigen Zufluss erhält jetzt das erste Bogengefäss durch ein sehr schwaches Gefäss, wchches aus der ventralen Verlängerung der ersten ausführenden Kiemenarterie entsteht, aber so dünn ist, dass man es nur mit Mühe bis zu seiner Einniundung in das erste Bogengefüss verfolgen kann.

\section{Stadium.}

O b e r f 1 ä c li e n b il d: Als IV. Stadium warle cine Larve von $16 \mathrm{~mm}$ Länge benutat, deren änssere Gestalt sehr charakteristisch ist, so dass sie sich gut von jüngeren und älteren unterscheiden lässt. Das Dotterorgan ist stark cingezogen, so dass seine Grenzen auf dem Oberflächenbild nicht deutlich hervortreten. Der Kopf ist nach vorn gestreckt und trägt noch am vorderen Ende die Saugscheibe, deren Näpfe aber von aussen nicht mehr sichtbar sind. Hinter ihr, in riemlicher Entfernmo vom Auge liegt die längliche Nasenöffnung. Die Grenzen des Auges sind bei der conservirten Larve nicht scharf, während Pupille und Iris sich gut abheben. Die Mundöffnung ist bedeutend weiter als früher and wird ventral durch den Unterkiefer abgeschlossen, dessen Spitze schon das ventrale Ende der Saugscheibe erreicht. Der Kiemendeckel ist hinten abgerundet, wird aber durch einen freien Saum verlängert, der z. T. noch den Ansatz der vorderen Extremität verdeckt. Ventral finden sich ebenso jederseits breite Hautfalten, welche den Kiemenraum unten abschliessen. Die vordere Extremität ist fast kreisförmig;, zeig't am freien Rand einen dünnen Saum und ist in der ganzen Fläche nach lateral convex gekrümmt. Por dem Dotterorgan, 
†eber die Entwicklung und morphologische Bedeutung' etc. 475

welches äusserlich nur noch durch dunklere Pigmentirung zu erkennen ist, liegt das Herz in fast derselben Richtung wie die Iä̈ngsachse. Die Seitenlinie, sowie die Myomeren sind durch Pigment markirt; der embroynale Flossensaum umgiebt noch die Hälfte des Körpers, ist aber nicht mehr gleichmässig breit, sondern an den Stellen der bleibenden unpaaren Flossen verbreitert, an den daxwischenliegenden schmaler; an der Analöffnung ist der Saum jetzt nicht mehr vorhanden. Die Flossenstrahlen kann man an der strichförmigen Pigmentirung und den Verstärkungen des Bindegervebes im Saum erkennen. Die Anlage der hinteren Extremität ist noch sehr klein und sitzt am hinteren Ende des Dotterorgans als flacher dreieckiger Wulst.

Ergebis der Serie. 1. Anlage des Spritzlochkan a les: Die vom Pharynx ausgehende Tasche beträgt jetzt etwa $1 / 3$ der Stärke der Rachenwand und ist ventral begrenzt durch einen Wulst, in welchem das dorsale Stïck des ersten Bogengefässes verläuft. Lateral stösst an die Tasche der iibrige Theil der Anlage, der noch strangförmig ist; besonders deutlich tritt jetzt das hohe cylindrische Epithel hervor, welches ebenso, wie in vorigen Stadium mit dem durch die Gehörkapsel kommenden Nerven Verbindung hat (Fig. 8).

2. Ki emenbogen: Die Kiemenbogen sind sämmtlich ausgebildet und auch alle vier von der Rachenwand losgetrennt; sie haben eine schwach S-förmige Krümmúng und besitzen zwei Reihen von Kiemenblättchen, ohne secundäre Fortsätze. Von Kiemen oder Kiemenanlagen am Kiemendeckel ist ansser dem beschriebenen Wulst ventral von der Spritzlochtasche nichts zu bemerken; die dort liegenden Gefässe verhalten sich auch gar nicht wie Kiemengefässe, sondern wie Ernährungsgefässe.

3. Herz und Gefässe. a) Herz: Das Herz hat annühernd die Lage wie beim erwachsenen Thier; es wird nur noch wenig vom Dotterorgan ventralwärts gedrängt. Man erkennt auf Querschnitten deutlich den Klappenapparat zwischen Atrium und Ventrikel; auch im Bulbus sind bereits einige Anlagen von Klappen vorhanden. Der Bulbus ist starkwandig, hat eine dicke Ringmuskulatur und biegt mit seinem binteren Theile nach der Seite aus, so dass sein Lumen anf dem Medianschnitt (Fig. $9 B u$ ) nicht ganz getroffen wird. Die Atrien bedecken von 
dorsal her den Bulbus und die vordere Hälfte des Herzens und legen sich seitlich dem Dotterorgan an.

b) Truncus: Der Truncus arteriosus zeigt sich gegen Stadium I-III stark verändert, was durch die Veränderung der Gestalt des Dotterorgans erklärlich wird. $\mathrm{Er}$ bildet zunächst nach dem Austritt aus dem Bulbus eine starke Erweiterung, von welcher nach hinten der gemeinsame Stamm für den dritten und vierten wahren Kiemenbogen abgeht; nach lateral zweigt sich das zweite zuführende Kiemengefüss ab (Fig. $10 \mathrm{Tr}$.), während der übrige Theil des Truncus nach vorn verläuft und sich nach kurzer Zeit in drei Aeste spaltet: Die beiden caudalwärts gelegenen sind die zufüilhrenden Arterien des ersten Kiemenbogens, das dritte zieht noch etwas nach vorn und ventralwiirts und theilt sich noch vor der Querebene, in welcher der hintere Angenrand liegt, in zwei Theile, welche symmetrisch auf die laterale Wand ubergehen und sich dann jederseits in dem unteren Theile des Kiemendeckels verzweigen (Fig. 9 u. 10 h. v.)

c) B o g.e n $g$ ef ä s s e: Die zuführenden Kiemengefüsse gehen in der oben beschriebenen Weise vom Truncus $a b$ und ziehen unterhalb des Knorpels der Kiemenbogen lateral- und dorsalwïrts, wobei sie sich allmählich in die Kiemencapillaren auflösen; sie überragen den Ansatz der Kiemenbogen an der dorsalen Rachenwand $\mathrm{um}$ ein ansehnliches Stück. Das Blut aus den Capillaren wird in dem ventralen Theile der Bogen von zwei Gefässen aufgenommen, welche unmittelbar unter dcm Knorpel verlaufen und sich im dorsalen Drittel zu einem Stamm vereinigen, der in doppelt S-förmiger Krümmung medialwärts umbiegend in die Aorta (Ao.) sich ergiesst. Das vierte ausführende Kiemengefäss vereiniot sich mit dem dritten (Fig. 9 u. 10), so dass nur drei zu- und drei ausführende Stämme vorhanden sind.

Das zuführende Gefüss der beiden ersten ursprüriglichen Bogen bestelit, wie schon gesagt, als ventrales Randgefäss, in dem anch noch ein Theil des zweiten Bogengefüsses steckt, weiter. Das dorsale Stück des ersten Bogengefässes ist unverändert vorhanden, erhält einen Zufluss ron der ventralen Verlängerung der A. efferens branch. I. und steht ausserdem mit dem ventralen Randgefäss in Verbindung durch ein reiches Capillarnetz. Der dorsale Theil des zweiten Visceralbogengefässes, der noch beim III. Stadium schwach vorhanden war, ist jetzt voll- 
Ueber die Entwicklung und morphologísche Bedeutung etc. 477

ständig verloren gegangen, so dass das dorsale Randgefäss des Kiemendeckels in seinem Hauptstamme nur mit dem ersten Bogengefässe in Verbindung (Fig. 9 u. 10 h. d.) ist, während es ausserdem mit dem ventralen Randgefüsse durch zahlreiche Capillaren communicirt.

d) Aorta und Carotis: Die Aorta ist, wie gesagt, in diesem Stadium unpaar; sie setzt sich aus den drei Stämmen der Kiemenarterien zusammen und läuft ventral von der Chorda rückwärts. Die Carotis entspringt aus der A. effer. branch. I., verläuft in ziemlich gerader Richtung nasalwärts und hat keine Anastomose mit dem entsprechenden Gefässe der anderen Seite. Sie theilt sich hinter dem Auge in zwei Aeste, von denen der eine sich dorsalwärts wendet und in kurzem Bogen nach vorn verläuft und die A. infraorbitalis darstellt, der zweite Ast vereinigt sich mit dem ursprünglichen ersten Bogengefässe zu einem starken, nach vorn ziehenden Stamme, welcher das Auge versorgt.

\section{Stadium.}

Oberflächenbild: Schon beim IV. Stadium war der Dottersack klein und von aussen kaum sichtbar; es wurde nun als nächstes Stadium ein solches gewählt, bei dem der Dottersack vollkommen verschwunden ist, die eigentliche embryonale Entwicklung also ihr Ende erreicht hat. Auf dieser Entwicklungsstufe ist die äussere Gestalt der des erwachsenen Thieres bis auf wenige Punkte gleich. Der Kopf allein misst jetzt etwa $1 / 3$ der ganzen Körperlänge, welche $26 \mathrm{~mm}$ beträgt. Einen grossen Theil des Kopfes wieder $(2 / 5)$ nehmen die schnabelartig verlängerten Kiefer ein, welche mit einer grossen Zahl kegelförmiger Zähne versehen sind. Das Vorderende trägt eine längliche Verdickung, welche in ihrem unteren Theile die ehemalige Saugscheibe darstellt und in ihrem obern Theile das Geruchsorgan trägt. Oberhalb des Oberkieferrandes erkennt man eine seichte Rinne und in derselben die Stellen der Hautsinnesorgane in Gestalt isolirt stehender Oeffnungen, die entweder paarig und dann rund, oder unpaar und langgezogen sind. Das Auge hat eine längliche Form, sein senkrechter Durchmesser ist kleiner als der horizontale und sein innerer Bau ist von dem beim erwachsenen Fisch kaum verschieden. 
Der Kiemendeckel ist gross und breit, trägt hinten und ventral einen freien Saum und seine hintere Begrenzung bildet etwa einen Kreisbogen; ventral vereinjgt sich der freie Saum beider Seiten und verschliesst die Rachenhöhle. Der übrige Körper ist schlank gebaut und wird allmählich nach dem Schwanze zu schmaler. Die Flossen sind bereits alle ausgebildet, der embryonale Flossensaum ist iberall verschwunden mit Ausnahme der Strecke zwischen hinterer Extremität und Analöffnung und ausserdem um das freie Körperende herum zwischen Rïcken- und Schwanzflosse. Das Schwanzende zeigt in diesen Stadium schon sehr dentlich die Heterocercie; hinter der Analöffnung spitzt sich das Schwanzende etwas zu und wird am Ansatz der Schwanzflosse plötzlich sehr schmal, so dass hinter derselben ein $3 \mathrm{~mm}$ langes, sehr schmales Endstück liegt, in welchem die Chorda und das Ende des Rückenmarkes enthalten ist. Die Seitenlinie-nimmt ihren Anfang am oberen Ansatze des Kiemendeckels und verlaiuft in fast gerader Richtung, durch Pigmentirung bezeichnet, candalwärts; sie erreicht die Schwanzspitze nicht, sondern endigt am dorsalen Ende des Ansatzes der Schwanzflosse. Die Flossen haben bereits starke Flossenstrahlen und sind gut von cinander zu unterscheiden. Die vordere Extremität hat schon annühernd ibre volle Grösse im Verhältniss zur Länge des Thieres und hesitzt in der dünnen distalen Hälfte Flossenstrahlen, während die hintere Extremität noch weiter in der Entwicklung zurückgeblieben ist und noch keine Strahlen, sondern nu einen feinen durchsichtigen Saurn zeigt.

Im durchfallenden Lichte crkennt man bis rum Vorderrand des schmalen Schwanzstïckes 66 Muskelscgmente, während dabinter noch undeutliche, nicht abgrenzbare, lïngsverlaufende Muskelfasern sichtbar sind.

Ergebniss der Serie. 1. Anlage des Sṕritzlochkauales: Der in Fig. 9 dargestellte Querschnitt ist so getroffen, dass auf der rechten Seite gerade das Ende der ganzen Anlage in einer Ausbuchtung der Gehörkapsel zn sehen ist, während anf der linken Seite die Mitte der pharyngealen Oeffnung erscheint; auf dieser Seite bemerkt man auch im Knorpel der Gehörkapsel den zur Spritzlochtasche binziehenden Nerven. Zur Orientierung in Bezug auf die Topographie der pharyngealen Oeffnung der Tasche dient die plastische Reconstruktion der 
Ueber die Entwicklung und morphologische Bedeutung etc. 479

Kiemengegend bei dem vorliegenden Stadium, welche Fig. 14 darstellt. Es ist reconstruirt: Der ganze ventrale Theil des Kopfes unterhalb der Gehirnbasis vom hinteren Rand des Auges bis zum Ende des Kiemendeckels; die Begrenzung in medialer Richtung geht bis an die Medianebene selbst. Das Modell ist beim Zeichnen mit dem dorsalen Theil etwas hintenïber geneigt, damit die Oeffnung. der Spritzlochtasche $\left(S p .{ }^{\prime}\right)$ in ihrer Grösse und Form deutlicher wird, da sie in der natürlichen Stellung von der Decke der Rachenhöhle zum grossen Theil verdeckt wird. Unmittelbar nnter der Gehirnbasis liegt die vorn zugespitzte Chorda; das vordere Ende des ersten Kiemenbogeus ist sichtbar, vom zweiten erscbeint nur ein ganz kleines Stück. Im Durchschnitt ist aússerdem getroffen das Her\% mit dem Atrium, das dorsal vom Ventrikel liegende Ende der Leber (He.), der Bulbus mit den Klappenreihen $(B u$.) und der Truncus, von dem die verschiedeuen zuführenden Kiemenarterien ausgehen. Die pharyngeale Oeffnung der Spritzlochtasche liegt etwa in der Querebene des Vorderendes der Chorda und hat ungefähr die Gestalt eines Kreises und einen Durchmesser von $0,2 \mathrm{~mm}$.

Der Spritzlochgang wird lateral schmaler, so dass auf Fig. 11 $S p^{\prime \prime}$ der hintere Theil des Ganges nur als kleine Oeffnung eben sichtbar ist. Ventral von der Tasche liegt ein starker etwa halbkreisförmiger Wulst, in dessen freiem Rande das ursprüngliche erste Bogengefüss zieht. Das Lumen desselben ist nach hinten zu bis zu seiner Umbiegung nach der Seite eröffnet (d. I.).

Der weitere Verlauf des Spritzlochganges muss auf der Serie verfolgt werdèn, da es nicht möglich wäre, ihn auf einem Schnitte ganz zu treffen. Die weite pharyngeale Tasche geht nur etwa bis zur Mitte der Rachenwandung und hat die Gestalt eines Trichters, dessen enges Ende lateral liegt. An diese Tasche schliesst sich unmittelbar als Fortsetzung ein viel engerer Gang an, der in seinern ganzen Verlaufe dieselbe Weite hat und sich in einer bogenförmigen . Krümmung: durch den Knorpel der Gehörkapsel bohrend, lateral-, dorsal- und rückwärts rieht, um in einer Aushöhlung des erwähnten Knorpels blind zu endigen; das Ende erreicht die Hant nicht, sondern ist durch Bindegewebe von ihr getrennt. Eine flache Einsenkung der Haut ist zwar sichtbar, wird aber wohl nur durch die Anshöhlung des Knorpels bedingt, 
da die Epidermis keine Veränderung der Zellform u. s. w. elleidet.

Der Nerv, dessen Abgang vom Gehirn resp. Ganglion in Fig. 11 R.o. auf der linken Seite sichtbar ist, durchsetzt den Knorpel in einem engen Kanal und tritt zu-der medialen Wand des engen Ganges, dessen Zellen an dieser Stelle hoch und cylindrisch sind, während die übrigen Wände das Epithel der Rachenhöhle zeigen.

2. Ki emenbogen: An den Kiemenbogen ist gegen das vorige Stadium nicht viel Nenes zn bemerken; sie sind gestreckter, vorn der Schädelbasis näher, als früher mul zeigen lange primäre Kiemenblättchen mit vereinzelten secundären Fortsätzen; dic Kiemengefüsse verhalten sich ebenso, wie bei erwachsenen Thieren. Merkwürdiger Weise ist selbst in diesem späten Stadinm weder ron einer Hyoidkieme, noch von ciner Psendobranchie das Geringste zu seben; das Gefäss, welches der zuführenden Arterie der Hyoidkieme bei erwachsenen Thieren entspricht, zieht unter dem Epithel der Rachenböhle und des Kiemendeckels fort, ohne dass man am Epithel oder an dem Gefüss irgend cinc Andentung fände, welehe anf die Bildung einer Kieme schliessen liesse (Fig. 11 h. v.).

Die Anlage der Spritzlochkieme dagegen ist auf diesem Stadiun bereits sichtbar; der ventral von der Spritalochtasche liegende Wulst wird, wie das spätere Stadium lehrt, in seinem hinteren Theile zur Anlage der Kieme.

3. Her\% und Gefäse. a) Herz: Das Herz ist gegen das vorige Stadium gestreckter und den des erwachsenen Fisches ausserordentlich ähnlich. Der Ventrikel hat schon die eigenthïmliche, einer dreiseitigen Pyramide entsprechende Gestalt, und seine Wände zeigen die später noch viel deutlicheren Trabckel. Die Atrien bedecken zu zwei Dritteln den Bulbus, der schon 4 Reihen Klappen besitzt.

b) Truncus: Der Truncus zeigt keinerlei auffallende Veranderung, nur ist er der Verinderung der Lage der Kiemenbogen entsprechend länger, und die zufülurenden Kiemengefüsse gehen im spitzen Winkel ron ihm lateral- und rïckwärts ab (Fig. 12 u. $13 T r$.)

c) B og eng ef ̈̈ sse: Ueber die Gefüsse der walıen Kiemen ist das Wichtigste bereits gesagt; sie rerhalten sich im Wesent- 
Ueber die Entwicklung und morphologische Bedeutung etc. 481

lichen nicht anders als im vorigen Stadium. Das Gefässstück, welches den gemeinsamen Stamm für die beiden ersten ursprünglichen Bogengefässe darstellt, ist etwas weiter nach vorn ausgewachsen, worunter zu versteben ist, dass sich die beiden Schenkel der Schleife, welche dieses Gefäss bilclet, verlängert haben; zugleich ist es verbältnissmässig dünner als im vorigen Stadium. Es verläuft noch im unteren Rande des Kiemendeckels und versorgt den ventralen Theil desselben (Fig. 12 u. $13 h$. v.).

Der ursprüngliche dorsale Schenkel des ersten ausführenden Bogengefässes hat sich entsprechend dem Wachsthum der Spritzlochtasche etwas verlängert. Wülırend noch im vorigen Stadium (Fig. 10 d. I.), das Gefäss in ziemlich gerader Richtung zwischen der Einmändung der ventralen Verlängerung des ersten ausführenden Kiemengefüsses und der Carotis median- und vorwärts zog, zeigt sich.jetzt, dass diese Strecke eine starke Knickung (Fig. 13 d.'I.) nach hinten erfahren hat; das Gefüss hat also seine Lage zu der Spritzlochtasche geändert, indem dieser Bogen ventral und mit dem Scheitel hinter die Spritzlochtasche zn liegen kommt. Es kann nach den vorliegenden Zeichnungen nicht zweifelhaft sein, dass man es immer noch mit dem ehemaligen ausfuihrenden Gefäss des ersten Bogens zu thun hat. An der Stelle, wo dieses Gefüss am meisten lateral liegt, mündet die ventrale Verlängerung des ersten Kiemengefässes ein, nachdem sie vorher an der vorderen Umbiegungsstelle verschiedene Zweige \%. B. für den Unterkiefer nach vorn n. s. w. abgegeben hat. In der Verlängerung des ursprünglichen ersten Bogengefässes nach hinten liegt das dorsale Randgefäss, welches mit dem ventralen Randgefäss zahlreiche Anastomosen hat. Von dem dorsalen Stück des zweiten Bogengefüsses ist nichts mebr zu sehen; in die Carotis münclen in diesem Stadium ausser dem erwähnten vor der Spritzlochtasche gelegenen keine bedeutenderen Aeste von lateral ein.

d) Aorta und Carotis: Die Aorta zeigt genan dieselben Verhältnisse, wie im vorigen Stadium; die beiden Aa. effer. branch. I vereinigen sich zu einem Gefäss, in welches die beiden vereinigten Aa. effer. branch. II und später die Aa. effer. branch. III und IV von der ventralen Seite einmünden (Fig. 12 und 13 Ao.).

In dem Verbalten der Carotis zeigen sich gegen Stadium IV keine Veränderungen. 


\section{Stadium.}

Oberflächenbild: Das älteste Stadium, welches mir für diese Untersuchmo'en zur Verfügung stand, hat bereits vollständig das Aussehen des erwachsenen Fisches. Es hat eine Länge von $113 \mathrm{~mm}$. Der Kopf ist vom Vorderende bis zum hinteren Rande des Kiemendeckels $33,3 \mathrm{~mm}$ lang, wovon allein auf die Kiefer 17,7, also über die Hälfte kommen. Der Kopf ist schlank gebaut und spitzt sich nach vorn ziemlich gleichmässig zu ; die Kiefer haben ihre endguiltige Gestalt erreicht und sind mit mehreren Reihen kegelförmiger Zälme besetat, in deren Acbse ein Stab differenter Substanz gelegen ist, der an der Spitze des Kegels in Form einer feinen Nadel frei hervorragt. Am Vorderende befinclet sich noch die in vorigen Stadium crwähnte Verdickung, jedoch olne eine Spur der Saugscheibe; diese Verdickung verlicrt sich nie, sondern ist beim erwachsenen Thier noch in derselben Weise sichtbar und trägt in ihrem hinteren Abschnitte das Gertuchsorgan mit zwei Ausführungsöffnungen. Oberhalb der Zahnreihe am Obcrkiefer, cbenso um das Ange herum und am Kiemendeckel liegen zahlreiche Oeffumgen von Schleimkanälen, welche bald rund, bald länglich sind. Das Auge ist ziemlich gross, seine einzelnon Theile sind aber nicht zu erkennen, da durch die Conservirung die Hornhaut midurchsichtig geworden ist. Der Kiemendeckel hat dieselbe Gestalt und auch verhältnissmüssig dieselbe Grösse wie beim erwachsenen Fisch, doch ist der freie Saum etwas breiter. Der iibrige Kürper ist schlank und zeigt sehr dentlich die einzehnen Muskelabschnitte, lïsst aber noch keine Schuppen erkennen. Alle Flossen sind mit Strahlen versehen und vollkommen von einander getrennt, der embryonale Flossensaum ist verschwunden ausser an dem letzten schmalen Stück des Körperendes, wo er sich noch bei Thieren von iiber $15 \mathrm{~cm}$ Länge erhält.

Ergebniss der Serie. 1. AnIage des Spritzlo chganges: Die pharyngeale Oeffnung des Spritzlochganges ist etwa kreisförmig und misst $0,1 \mathrm{~mm}$. Diese Oeffnung entspricht aber nicht dem durchschnittlichen Lumen des Ganges, sondern ist bedeutend kleiner, da sich an der pharyngealen Oeffnung eine dęutliche Verengerung findet. Lateral von dieser engen Stelle erweitert sich der Gang etwas, sein Querschụitt ist von oben nach 
Ueber die Entwicklung und morphologische Bedeutung etc. 483

unten zusammengedrückt. $\mathrm{Er}$ zieht sich nun mit constantem Lumen von vorn und medial lateral- und rüekwärts, nur wenig dorsal ansteigend unterhalb der Kapsel des Gehörorgans. Kurz vor seinem blinden Ende zweigt sich dorsalwärts ein etwas schmalerer Gang ab, welcher die Gehörkapsel durchbohrt und dicht unterhalb der äusseren Haut in unmittelbarer Nähe eines Hautsinnesorganes endigt. Das blinde Ende des unter der Gebörkapsel liegenden Ganges bleibt ein bedentendes Stïck von der äusseren Haut entfernt.

Die beiden Gänge unterscheiden sich von einander durch die Form ibres Epithels; während der ventrale Theil der ganzen Anlage d. h. das gemeinsame Anfangsstück und die ventrale Fortsetzung dasselbe Epithel besitzt wie die Rachenböhle, findet sich an der ganzen medialen Wand des dorsal abgehenden Ganges ein hohes cylindrisches Epithel, theils in grossen Stücken, theils in ganz kleinen hügelförmigen Abtheilungen, die durch Spalten von einander getrennt sind; die laterale Wand wird von denselben Epithel bedeckt, wie es der übrige Theil der Anlage zeigt.

Der Nerv, welcher zu dem dorsalen Abschnitt des Ganges geht, tritt in derselben Querebene, in welcher anch die Mitte der pharyngealen Oeffnung liegt, aus dem Ganglion heraus und zieht in einem Kanal durch die Gehörkapsel lateral- und rüickwärts za der vorderen Seite des dorsalen Ganges, um von dort aus das hohe Epithel mit Nervenfasern zu versorgen. Ein kleiner Zweig dieses Nerven versorgt das erwähnte Hautsinnesorgan.

2. Kiemenbogen: Schon beim V. Stadium war der Bau der Kiemenbogen dem bei ausgewachsenen Fischen sehr ähnlich, bei diesem letzten sind auch bereits die secundären Kiemenblättchen vollkommen ausgebildet, so dass die vier wahren Kiemenbogen sammt ihren Kiemen in ihrem Bau und ihrer Versorgung genau dieselben Verhältnisse zeigen, wie bei den erwachsenen Thieren.

Deckelkiemen. a) Hyoidkieme: In diesem Stadium finden sich 'nun alch die beiden Deckelkiemen, die Hyoidkieme und die Pseudobranchie Johannes Milller's :ausgebildet. Das vorlere, ventrale und im Sinne des Blutstromes proxinale Ende der Hyoidkieme liegt etwa in der Mitte der seitlichen Rachenwand and in derselben Querebene, in welcher die 
zweite zuführende Kiemenarterie vorn umbiegt, um auf den Kiemenbogen überzugehen. Von dort zieht sich die Kieme, deren vordere Blättchen ventral gerichtet sind, in einem flachen Bogen nach hinten und nur sehr wenig dorsalwärts und biegt dann in ihrem hinteren Abschnitte stark nach dorsal, um etwa in der Höhe der Schädelbasis zu endigen. Der Abstand zwischen Anfang und Ende der Kiemen betrïgt in der Projektion auf die Horizontalebene gemessen $1,7 \mathrm{~mm}$. Die Kiemenblättchen gehen von der Convexität des bogenförmigen Ansatzes aus, also ventralund rïckwärts, und haben bis an die Spitze heran secundäre Blättchen.

b) Psendobranchie: Die Psentobranchic sitzt weiter dorsal und hält sich in ihrer ganzen Länge ziemlich anf derselben Höhe wie die Schädelbasis. Sie beginnt weiter hinten als die Hyoidkieme und reicht nicht so weit nach hinten wie jene. Thr Anfang liegt hinter der pharyngealen Oeffnung des Spritzlochganges und etwa in derselben Querebene wic der Anfang des dritten Kiemenbogens. Ihr Hinterende ist von dem dorsalen Ende der Hyoidkieme durch einen Zwischemraum getrennt; die Enden der beiden Kiemen berühren sich also nicht, wic bei den erwachsenen Thieren, sondern auf Schnitten, welche das Hinterende des Ansatzes der Hyoidkieme treffen, ist nichts melir von der Psendobranchie zu sehen. Die Blättchen der Psendobranchie sind dorsal- und rückwärts gerichtet und haben ebenfalls wie dié der Hyoidkieme secundäre Blättchen, welche von Kiemencapillaren durchzogen werden. Beide Kiemen liegen noch inncrhalb der geschlossenen Rachenhöhle und erreichen den freien Theil des Kiemendeckels nicht.

3. Herz und Gefässe: In Berug auf Herz, Truncus und Gefässe der vier wallen Kiemen ist keinerlei Unterschied im Bau gegen das Verhalten bei erwachsenen Thieren vorhinden, so dass ich ihre Beschreibung itbergehen kann.

Dagegen hat sich der Verlauf der beiden ersten Bogengefässe so verändert, dass eine genauere Besprechnng nöthig ist. Eine Reconstructionszeichnung ist von diesem Stadium nicht angefertigt, weil die Verhältnisse denen beim erwacbsenen Fisch so ähnlich sind, dass ich die Zeichung aus der Arbeit von Joh. M üller (Cit. 2) entlehnen kann.

Der ursprüngliche gemeinsame Stamm der beiden ersten 
Ueber die Entwicking und morphologische Bedeutung etc. 485

Bogengefässe (Stad. V. Fig. $12 h . v$.) ist Hoch vorhanden und verläuft noch in derselben Weise, wie früher vom 'Truncus zur lateralen Rachenwand. Seine Fortsetzung, das frübere ventrale Randgefäss ${ }^{1}$ ), ist jetzt zum zuführenden Gefäss der Hyoidkieme geworden, A. afferens hyoidea (Fig. 15 aff. $h$.) Das Blut aus den Capillaren derselben nehmen verschiedene kleine Gefüsse anf, die dorsal von der Kieme sich za einem Stämmchen vereinigen, welches sich allmählich von der Kieme nach vorn entfernt. Weit vor dem Anfang derselben vereinigt es sich mit dem Gefässe, welches aus der ventralen Verlängerung der A. effer. branch. I. entsteht (Fig. 15 br.o.) und in der seitlichen Rachenwand dorsalund rückwärts zieht. Das ans ihnen resultirende Gefäss (Fig. $15 a f f . s p$.) zieht zuerst ausserhalb der Knorpel der seitlichen Rachenwand nach vorn und geht dann in einem Bogen um den Knorpel herum an die innere Seite der lateralen Rachenwand nach hinten. Dieser letate Abschnitt des Gefässes dient als zuführendes Gefäss für die Pseudobranchie; deren Lage bereits S. 484 beschrieben ist. Das Blut ans den Capillaren dieser Kieme wird von einem Gefüsse, welches parallel dem zuführenden zieht, aufgenommen; letzteres ist umgekehrt, nach vorn gerichtet und durchläuft den Wulst, welcher ebenso wie im vorigen Stadium die pharyngeale Oeffnung des Spritzlochkanales ventral begrenzt; sodann wendet es sich allmählich medianwäirts und vereinigt sich mit der Carotis. Die Versorgung des Kiemendeckels geschieht nicht mehr durch die Randgefässe, sondern auf dieselbe Weise, wie beim erwachsenen Thier.

\section{Zusammenfassung.}

1. Spritzlochtasche und -gang: Aus den Untersuchungen geht hervor, dass bei Lepidosteus in keinem Entwicklungsstadium ein Spritzloch, d. h. eine äussere Oeffnung der Hyomandibularspalte vorkommt, wie das bei den in der Literatur (Cit. 3 und 5) beschriebenen Stadien bereits bekannt war. Es bildet sich nur der pharyngeale Theil der Spalte zu einem schräg lateral-, dorsal- und rïckwärts verlaufenden Gange aus. Anffallend jst besonders das späte Auftreten desselben, da er seine grösste

1) Dieses „ventrale Randgefäss" enthält, wie aus den früheren Stadien herrorgeht, das Anfangsstück des zweiten Bogengefïsses. 
Ausbildung zu einer Zeit erreicht, wo die embryonale Entwicklung vollkommen zu Ende ist, während man erwarten sollte, dass er sich gleichzeitig mit den ïbrigen Spalten entwickelt.

Zur Zeit des Ausschlüpfens besitzt die Larve bereits zwei Kiemenbogen, die aber noch nicht von einander getrennt sind; durchgängig ist nur die erste Kiemenspalte zwischen Hyoid- und erstem Kiemenbogen; weder an der äusseren noch an der inneren Rachenwand ist vor der ersten Kiemenspalte irgend eine Andeutung von einer Spaltenbildung zu sehen. Erst auf Querschnitten (Fig. 1) erkennt man die Anlage als einen soliden Zellstrang, der bis an die unveränderte Epidermis reicht.

Während sich nun in den nächsten Stadien, entsprechend dem Verbrauch des Dotters, die wahren Kiemen schnell und vollkommen ausbilden, macht die Entwicklung des Spritzlochganges nur geringe Fortschritte. Sehr langsam stülpt sich vom Rachen her das Epithel ein, während der pharyngeale Theil des Zellstranges sich trichterförmig erweitert. Zugleich ändert sich die Richtung des Stranges, er erreicht schon vom 3. Stadium an nicht mebr die Epidermis, sondern ist durch Bindegewebe von ihm getrennt, verlängert sich aber dorsalwärts, so dass er einen Bogen um den Boden der Gehörkapsel herum beschreibt. Mit dieser Veränderung der Richtung tritt anch eine differente Weiterentwicklung der Zellen auf. Während der laterale, ventrale Theil des Stranges ebenso wie die Wände der trichterförmigen Oeffnung dasselbe Epithel wie die Rachenhöble zeigt, entwickelt sich aus dem medial liegenden Theil des Zellstranges cine Schicht eines hohen cylindrischen Epithels. Sehr allmählich geht nun die Weitereröffnung der Anlage vom Pharynx her vor sich, so dass erst der ventrale Theil sich offnet und dann der dorsalwärts ausgewachsene Strang. Die Gehörkapsel, welche in den vier ersten Stadien medial vom Strange liegt, sich ihm aber immer mehr nähert, wächst langsam um ihn herum, so dass nachher der dorsale Theil des Spritzlochganges in einem Kanal der Gehörkapsel liegt; sein blindes Ende ragt dorsal aus dem Knorpel hervor. Ihre vollständige Ausbildung erreicht die Spritzlochanlage erst lange nach dem Ende der embryonalen Entwicklung; sie findet sich noch bei dem ältesten Stadinm. Der ventrale Theil des Ganges ist noch etwas weiter lateralwärts ausgewachsen, so dass der dorsale engere Kanal nicht mehr am Ende, sondern 
Ueber die Entwicklung und morphologische Bedeutung etc. 487

weiter medial einmündet. Der ganze ventrale Theil der Anlage ist mit demjenigen Epitbel, welches auch die Rachenhöhle hat, ansgekleidet. An der dorsalen Abzweigung findet es sich dagegen nur an der Jateralen Wand, während die mediale von dem erwähnten cylindrischen Epithel eingenommen wird. Weder der ventrale noch der dorsale Theil des Kanales erreicht die äussere Haut, beide sind durch Bindegewebe von ihr getrennt.

In welcher Weise sich die Rückbildung des Spritzlochganges, der sich bekanntlich beim erwachsenen Thier nicht vorfindet, vollzieht, war mir nicht möglich, zu entscheiden, da das älteste Stadium der jüngeren Thiere (Stad. VI) noch den Gang besitzt.

Der Nerv, dessen Beziehungen zu dem Cylinderepithel des Ganges bei älteren Stadien leicht nachzuweisen sind, R. oticus, nach Wright $(R . o$.), findet sich in allen früheren Stadien auch. Beim jüngsten Embryo scheint jedoch seine Verlaufsrichtung gerade entgegengesetzt der bei älteren; man findet dort einen Nerven, der von hinten her zn dem Zellstrange zieht und an dem lateralen Ende desselben an die Zellen herantritt (Fig. 1) und dort endigt; denn auf den weiter vorn grelegenen Sclonitten ist nichts mehr davon zu sehen. Leider ist der Uebergang zwischen den beiden Verlaufsarten nicht zu constatiren, da das II. Stadium, auf welches es dabei ankäme, so wenig differente Zellen hat, dass man den Nerven nicht herausfindet. In dem nächsten Stadium zieht er bereits in derselben Richtung, wie die Spritzlochanlage und tritt an das dorsale Ende des Stranges.

Diese Richtung behält er bis zuletzt bei und man kann in dem letzten Stadium seine Fasern bis zu den cylindrischen Zellen der medialen Wand verfolgen. Ein kleiner Ast des Nerven geht, wie im letzten Stadium sichtbar ist, und wie anch $W \mathrm{r}$ ig $\mathrm{t}$ beschreibt, zu einem dicht dabei liegenden Hautsinnesorgan.

B a I f o r und Parker (Cit. 3) beschreiben den Spritzlochgang von einem Embryo von ungefähr $10 \mathrm{~mm}$ Länge, der fast genau meinem I. Staḍium entspricht. Sie erwähnen richtig, dass in diesem Stadium mehr von einer doppelten Entodermlage, als von einer wirklichen Tasche zu sprechen sei (Cit. 3, Taf. 24, Fig. 43), und heben als bemerkenswerth hervor, dass der Gang selbst beim Embryo von Lepidosteus rudimentair sei. Die Bemerkung dieser Autoren, dass der Gang selbst keine weitere Entwicklung erfalıre, ist nach dem, was ich mitgetheilt habe, 
nicht zutreffend; noch weniger die Angabe, dass derselbe kurz nach dem Ausschlüpfen schwinde. Diese irrthümliche Erklärung. lïsst sich vielleicht auf dic grosse Schwierigkeit zurïckfübren, in dem zunächst folgenden Stadium (Stad. II, S. 470) den Strang zu erkennen; auch ich war anfünglich in Gefahr, denselben ganz zu übersehen und konnte iln erst bei erneuter Untersuchung, nachdem ich ihn bei jüngeren und äteren Stadien geschen hatte, auf ganz dïnnen Schnitten auffinden. Eine sehr wesentliche Erweiterung haben diese Angaben $B$ a 1 f o n r's und Parker's erfahren durch die interessante Entdeckung des dorsalen Divertikels und seines Neuro-Epithels von Wright, worauf an anderer Stelle (S. 496) Bezug genommen ist.

2. Ki emenbogen und Ki emen: Zn der Zeit, wo die Larve das $\mathrm{Ei}$ verlässt, sind noch keine zur Respiration tallslichen Kiemen vorhanden. Es sind ïberhaupt erst \%wei Kiemenbogen angelegt, welche aber noch nicht einmal von cinander getrennt sind, nur die erste Spalte geht durch die ganze Wand lindturch. Die Bogen haben noch keine Blättchen und die Gefässe gehen, ohne Capillaren zu bilden, hindurch (S. 468). In demselben Maasse aber, wie das Dotterorgan schwindet, entwickeln sich auch die Kiemen und sehon im II. Stadium, bei einer Larve mit noch sehr grossem Dottersack (S.471) finden sich drei wohlausgebildete Bogen mit primären Kiemenblätchen, und auch der vierte ist bereits angelegt; sehr bald (Stad. III, S. 474) wird auch der vierte Bogen durch das Auftreten der letzten Spalte abgesetzt und erhält Blättchen. Während der ubrigen cubryonalen Zeit verlängern sich die Bogen und erhalten demgemäss eine grössere Zahl primärer Blättchen, die secundären finden sich anch nach dem Verbrauch des Dotters erst schwach angedeutet (Stad. V, S. 480) als kleine rundliche Fortsätze an einigen primäien Blätchen. Erst in der Zeit, wo die Thiere fast vollstaindig ansgebildet sind und ganz die Gestalt der erwachsenen zeigen, treten auch die secundären Blättchen auf.

Deckelki em e n: Die beiden Deckelkiemen erscheinen seltsamer Weise erst, wenn die gesammte embryonale Entwicklung beendet ist. Während man erwarten sollte, wenigstens die Anlage schon bei frühen Stadien zu treffen, ist, solange die Larven noch Dotter haben, keine Spur davon zu sehen. Nach Verbrauch des Dotters erkennt man zuerst die Aulage der Psendo- 
Ueber die Entwicklung; und morphologische Bedeutung etc. 489

branchie als kleinen Wulst ventral von der Spritzlochtasche, welcher von dem späteren Kiemengefässe durchflossen wird. Er zeigt keine Verïnderung: des Epithels, sondern erscheint als einfache mit Bindegewebe gefüllte Auftreibung der Rachenwand. Bei demselben Thier sieht man aber von einer Anlage der Hyoidkieme noch nichts; die Rachenwand ist an der Stelle, an welcher später die Kieme sit»t, noch ganz glatt und das Gefüss besitzt auch keinerlei Besonderheiten, die es als Kiemengefüss erkennen liessen. In der folgenden Zeit entwickeln sich beide Kiemen allmählich und sind beim VI. Stadium fast vollständig. vorhanden; sie besitzen primäre und secundäre Blättchen und ganz vollkommene Kiemencapillaren, machen also beide den Eindruck vollständig entwickelter Kiemen. Die Hyoidkieme ist bedeutend länger als die Pseudobranchie und reicht bis in die Nähe des Endes der letzteren.

2. Gefäs se der Vis ce ra lbogen: Beim Ausschlïpfen der Larve sind, wie bereits erwähnt, erst vier von den sechs primitiven Bogengefassen vorhanden, welche zu je zwei mit einem gemeinsamen Stamm aus dem Truncus entspringen (Fig. 3). Die beiden candalwärts gelegenen sind die Gefässe fur den crsten und zweiteu Kiemenbogen, Von den beiden vorderen zieht das eine vor, das andere hinter der Anlage des Spritzlochkanales vorüber. Alle vier ergiessen ihr Blut in einen längsverlaufenden Stamm, der sich mit dem entsprechenden der anderen Seite linter den' Kiemenbogen vereinigt und die Aortenwurzel darstellt. Das zweite Bogengefüiss (Fig. 2 u. 3 arc. II) hat eine Verbindung mit dem ersten (Fig. 2 r. 3 arc. $I$ ) und giebt ein ventrales Randgefüss (Fig. $3 h . v$.) ab, welches mehrere Schleifen im Kiemendeckel bildet, die offenbar zur Ernährung dienen.

Während sich nun allmählich (Stad. II u. III) die anderen beiden wahren Kiemengefässe in der typischen Weise ausbilden (Fig. 5 u. 6), machen die beiden ersten Bogengefässe sehr starke Veränderungen durch, so dass ihr eigentlicher Charakter als Bogengefässe verschwindet. Schon nach sehr kurzer Zeit (Stad. II, Fig. 5 u. 6) wird die Verbindung zwischen dem gemeinsamen zuführenden Stamm und den beiden dorsalen Aesten nicht mehr durch die starken ursprünglichen Bogengefässe bewirkt, sondern durch eine grössere Anzahl feinerer Aeste, ist also fast capillär geworden. Sehr deutlich sind noch vorhanden 
der gemeinsame Stamm und die dorsalen in die Carotis einmündenden Stücke, welche dic Anlage des Spritzlochganges zwischen sich fassen. Die beiden dorsalen Stücke sind ausserdem jetzt durch eine Anastomose verbunden und nach hinten hat sich an dem zweiten Bogengefüss das dorsale Randgefäss des Kiemendeckels entwickelt. Auch die beiden Randgefäisse stehen mit einander durch Capillaren in Verbindung.

Das dorsale Stiick des zrveiten Bogengefässes findet sich in spüteren Stadien überhaupt nicht mehr, es geht schon sehr bald (Stad. IV) zu Grunde (S. 476). Im III. Stadium ist es nur noch mit Mühe erkennbar, während man im IV. keine Spur mehr davon entdeckt; man sieht dann nur noch ein Gefäss von der Seite kommend in die Carotis münden und dieses liegt vor der Anlage des Spritzlochganges; es ist das erste Bogengefiiss (Fig. 9 u. 10 d. I.). Die Anastomose zwischen den beiden ersten Bogengefässen hat sich stärler ausgebildet, und clas dorsale Randg'efäss ergiesst jetzt sein Blut durch das' erste Bogengefäiss in die Carotis.

Nach der Anordnung der Gefässe im II. Stadium erhält die Carotis ihr Blut einerseits aus den Kiemenarterien, welche ausscrdem noch den ganzen Körper zu versorgen haben, und anderseits durch die capillare Verbindung von den ventralen Randgefässe Blut aus dem Her\%en, welches in diesem Stàdium bei der verhältnissmässigen Grösse des Dotterorgans wohl in den Dottervenen arteriell gemacht wird; es erhalten anf diese Weise die wichtigen Organe des Kopfes hellrothes Blut. Mit den Schwinden des Dotterorgans und der Ausbildung der Kicmen iibernehmen natürlich die letzteren die Arterialisirung des Blutes, and es tritt desbalb eine besondere Verbindung "zwischen den ausführenden Kiemenarterien und dem dorsalen Theil des ersten uisprünglichen Bogengefässes auf; es ist dies dasjenige G'efüss, welches die Fortsetzung der ventralen Verlängerung der ersten ausführenden Kiemenarterie bildet; es ergiesst sein Blut lateral in das erste ursprïngliche Bogengefüss (Fig. 9 u. $10 \mathrm{br}$. o.). Die Verbindung zwischen dem ventralen und dem dorsalen Randgefäss bildet ein sehr reichliches, über den ganzen Kicmendeckel verbreitetes Netz von Anastomosen und Capillaren. Die Existenz so starker Ernährung:sgefässe für den Kiemendeckel wird erklärt durch das gewaltige Wachsthum des letateren; denn in späteren 
Ueber die Entwicklung und morphologische Bedeutting etc. 491

Stadien (Stad. VI) findet man sie nicht mehr, sondern sie werden durch andere kleinere Gefässe ersetzt, da dann der Kiemendeckel nur noch in demselben Maasse weiter wächst, wie die übrigen Organe.

Eine bedeutsame Veränderung bemerkt man auch im Verlaufe des ursprünglichen ersten Bogengefässes (Stad. V, Fig: 13 d. I.). Während es früher (Stad. IV, Fig. 10 d. I.) in fast gerader Richtung zur Carotis medianwärts läuft, bildet es allmählich einen nach vorn concaven Bogen, d. h. seine Mitte verschiebt sich nach hinten, sodass jetzt nur noch Anfang und Ende vor der Anlage des Spritzlochganges liegen, wäbrend die Mitte sich ventral von der, pharyngealen Oeffnung des Ganges nach. hinten zieht. Diesés Verhalten bildet den Uebergang zum letzten Stadium, in welchem sich die Gefässe im Wesentlichen wie beim erwachsenen Fisch verhalten (Fig. 15 br.o.). Das Gefüss, welches aus dèr ventralen Verlängerung des ersten Kiemengefüsses hervorgeht, ist die A. hyoideo-opercularis Joh. Miiller's. Sie vereinigt sich mit dem ansführenden Gefässe der Hyoidkieme (s. 11. S. 499) und bildet mit ihm zusammen den Anfangstheil des ursprïnglichen ersten Bogengefüsses; letzterer zieht in cinem vorn convexen Bogen ventral vom Spritzlochgang unter der Rachenschleimhaut nach hinten zn der inzwischen ausgebildeten Pseudobranchie, wo er sich in Capillaren auflöst; unmittelbar daneben liegt ein in entgegengesetzter Richtung verlaufendes Gefäss, welches in ziemlicher Entfernung von der pharyngealen Oeffnumg des Spritzlochganges medianwärts zur Carotis läuft und sich mit ilur vereinigt. Das ursprüngliche erste Bogengefäss macht also vom IV. Stadium an folgende Wandlungen durch: Betrachtet man die Strecke zwischen der Einmündung der Hyoideo-opercularis und der Vereinigung mit der Carotis, so ist im Stadium IV (Fig. 10 d. L.) die Verbindung geradlinig, beim Stadium V (Fig. 13 d. $l$.) ist sie ein nach rorn concaver Bogen und bei Stadium VI, wie beim erwachsenen Thier ist dieser Bogen lang nach hinten ausgezogen, zwischen den beiden Schenkeln bilden Capillaren die Verbindung und aus dem urspruinglichen ersten Bogengefässe sind das zuführende und das ausführende Gefüss der Pseudobranchie geworden (Fig. 15 aff. sp. u. eff. sp.).

Die Hyoidkieme entsteht an dem chemaligen ventralen Randgefäss, welches das zuführende Gefüss für die Kieme wird. 
Das Blut wird dann von einen aus mehreren Aesten sich zttsammensetzenden Gefüss gesammelt, welches, wie oben (S. 491) gesagt, zu der A. hyoideo-opercularis geht und sich mit ihr vereinigt. Die beiden Randgefüsse verlieren, wie erwähnt, ibre Bedeutung und sind nicht mehr in diesem Stadium sichtbar.

\section{Morphologische Dentung und Literatur.}

Ueber die morphologische Bedentung des Spritzlochganges kann ein Zweifel nicht bestehen und bestcht anch in der That nicht in der Literatur.

Es kömute nur ein Punkt zur Erörterung Veranlassnng. bieten, welcher jelloch zu der Aufgabe meiner Arbeit in keiner direkten Beziehung steht, nämlich der dorsale Recessus (Otic diverticle von Wright). Es könnte sich nämlich fragen, ob diese Ausstiilpung als dic eigentliche Fortsetzung des Spritzlochganges; die dorsalwirts abgebogen ist, oder als cine sccundäre Bildung aufzufassen sei. Im Voransgrehenden ist das Gebilde mehrfach erwähnt worlen; die verabeiteten Stadien waren jedoch nicht geeignet, dic Entwicklung desselben in der genïgenden Weise Sehritt für Schritt \%u verfolgen. Doch ist es mir nach allem wahrscheinlich, dass die $W \mathrm{l}^{\circ} \mathrm{g}$ h t'sche Ansicht die richtige ist. Indessen ist dies, wie gesagt, ein Punkt, del die unnittelbare Aufgabe ler Arbeit nicht berïhrt.

Anch über die Hyoillieme und ibre znfiilhrencle Arterie kann eine Unklarheit nicht bestehen; jedoch sind hier zwei Punkte erwähnenswerth, welche mit Ruicksicht auf die Psendobranchic und ihre Gefässe Bedeutung haben. Erstens nätnlich liegt die Kieme dem Hyomandibnlare an, hat also ihre Lage zu dem Skelet des Hyoidbogens bewahrt und ist nicht der ans iliesen Bogen hervorgehenden Hantfalte (dem Kiemendeckel) ge. folgt. Diese Thatsache gewinnt eine scharfe Beleuchtung durch den Vergleich mit Acipenser, wo die Hyoidkieme mit der Ausbildung des Kiemendeckels verschoben und in der Nähe des Hinterrandes des letzteren verblieben ist; die Kieme liegt bei Lepi dosteus an der Basis des Kiemendeckels. Dieses Lageverhältniss gewinnt Bedeutung für die Umgestaltung der im Bereiche des zweiten Bogengefässes während des IV. und V. Stadiums entstelıenden Capillarnetze. Indem nämlich die Kieme im Anfange desven tralen Randgefässes angelegt wird, wird der da- 
Ueber die Entwicklung und morphologische Bedeutung etc. 493

hinter liegende Theil des letrteren sammt den nach der dorsalen Seite emporführenden Capillarnetzen und dem dorsalen Randgefäss selber von der Blutzufulı abgeschnitten und damit das zeitweilig so reich entwickelte System des zweiten Gefässbogens zum grössten Theil dem Untergange iiberliefert. Die reiche und eigenartige Entwicklung des zweiten Gefässbogens besteht, wie im Stadium V geschildert wurde, darin, dass sich aus seinem Anfangs- und Endstiick das dorsale und ventrale Randgefüss des Kiemendeckels entwickelt, welche durch cin ausgedehntes Netz von Capillaren verbunden sind. Mit diesen Beziehungen des erwähnten Gefässgebietes als eines nutritiven Netzes, welches während der Periode des starken Wachsthums des Kiemendeckels bestehen bleibt, hängt wohl auch zusammen, dass die Kieme erst nach der Ausbildung des Deckels angelegt wird, denn irgend eine räumliche Behinderung für die Entstehung derselben liegt nicht vor.

Zweitens reicht am erwachsenen Thier das vordere (ventrale) Ende der Hyoidkieme nicht bis an das ventrale Ende der ersten Kiemenspalte, sondern bleibt, wie mir Herr H. Virchow mittheilt, bei eincr Kopflänge von $225 \mathrm{~mm} \mathrm{um} 17 \mathrm{~mm}$ von demselben entfernt. Dies Verbalten ist an sich am erwachsenen Thiere morphologisch nicht erklärbar, da es zwei Möglichkeiten für die Entstehung dieses Verhaltens g:iebt; erstens, class die Kieme sich verschoben hat, zweitens, dass das ventrale Ende derselben oblitcrirt ist. Die Entwicklang der Gefässe jedoch, wie sie im Vorausgehenden geschildert worden ist (Stad. I, Fig. 3) liefert hierfür die Erklärung, indem die Theilungsstelle für das erste und zweite Bogengefüss in früheren Stadien nicht in der Nähe der ventralen Mittellinie, sondern weiter seitlich gelegen ist, an ciner Stelle, welche dem ventralen Ende der Kieme entspricht. Da also hier erst, was nach $\mathrm{Sch}$ ch u d des ventralen Endes des ersten Bogengefüsses allerdings nicht mehr erkennbar ist, das eigentliche zweite Bogengefäss beginnt, so kann auch die Kieme nur bis an diesen Punkt heranreichen.

Die Rolle einer ausfülrenden Hyoidarteric wird nach einander von drei verschiedenen Gefässabschnitten ïbernommen: zuerst, bevor die Kiemen auftreten, von dem dorsalen Stiick des zweiten Bogengefüsses selber' (Stal. I u. II, Fig. 2, 3, 5, 6), dann, nachdem dieses fribzeitig zu Grunde gegangen ist (Stad. IV), 
von einer in der Verlängerung des dorsalen Randgefässes nach vorn in den ersten Gefässbogen führenden Anastomose, welche zwar schon früher vorhanden ist, jetzt aber erst eine erhöhte Bedeutung gewinnt. Endlich drittens, nachdem diese Anastomose im Verfolg der vorhin geschilderten Verödung des opercularen Capillargefüssnetzes gleichfalls verloren gegangen ist, durch eine mehr ventral gelegene Anastomose vom ventralen Randgefässe zur A. branchio-opercularis ${ }^{1}$ ); diese Anastomose entwickelt sich erst beim Entstehen der Kieme.

Eine wichtige morphologische Frage ist es, ob in einer der beiden zuletzt genamiten Verbindungen ein Gefäss gefunden werden kann, welches der in Seitenlinie gelegenen A. efferens hyoidea der Selachier entspricht, welche ja in dieser Klasse direkt und ausschliesslich die $A$. afferens spiracularis liefert. Ich kann in dieser Beziehung nur sagen, dass für keines der beiden Gefüsse cine ummittelbare Evidenz angeführt werden kann, dass aber $\%$ Gunsten des dorsalen das frïhe Auftreten und seine ziemlich seitliche Lagerung (Stad. II, Fig. 6), sowie die Richtung spricht und dass anch, wenn die Kieme fribzeitig genng ansgebildet wïrde, bevor das Gefäss zu Grunde geht, las letztere die norphologiseh geforderte Lage zur Hyoid-Kieme haben würde. Das zuletzt grebildete der beilen Gefässe, d. h. die letzte oder definitive A. efterens hyoidea, scheint mir keinen Anspruch darauf zu haben, mit der erwähnten A. efferens hyoidea der Sclachier homologisirt zu werden.

Psendobranchie und ihre Gefässe: Ich komme jetzt zu dem eigentlichen Problem meiner Arbeit, nümlich zu der Frage der morphologischen Natur dẹ Pseudobranchie, d. h. zu der Frage, ob die Pseudobranchie als Spritzlochkieme oder als cin Stïck der Hyoidkieme aufaufassen sei.

Hierfiul sind in der Literatur drei Punkte in Betracht gezogen worden: die Beziehung der Kieme zu der Spritzlochtasche, zu den Nerven und zu den Kopfgefüissen, wozu wolıl als vierter Punkt, gewissermaassen halb unbewusst, die Formverhältnisse der Kieme selbst hinzutreten.

1) Mit dem Namen A. branchio-opercularis bezeichne ich das Gefïssstück aus der ventralen Verlüngerung der A. efferens branchialis I., welches in der seitlichen Rachenwand zieht, vor dem Eintritt der A. hyoideo-opercularis, mit der es die A. afferens spiracularis bildet. 
Ưeber die Entwicklung und morphologische Bedeutung etc. 495

Letzteres möchte ich vorher berühren; die Pseudobranchie gleicht, wie Joh. M ïller deutlich hervorhebt und abbildet (Cit. 2, Tafel V, Fig. 6) genau der Hyoidkieme, und da beide sich mit ihren Enden berühren, so kann allerdings der unmittelbare Eindruck gewissermaassen mit Evidenz dafür sprechen, dass man es mit Theilen ein er Kieme zu thun hat. Dieser Eindruck kann für den Kenner des Selachierkopfes sich verstärken, indem bei der Mehrzahl von diesen die Spritzlochkieme nicht allein klein, sondern auch von rudimentürem Charakter ist, woraus, wenn man die Selachier als Vorlüufer der Ganoiden betrachtet, geschlossen werden könnte, dass, wenn die Spritzlochkieme bereits bei den Selachiern ihren Kiemencharakter nicht mehr constant aufrecht erhält, sic dann bei den Ganoiden wohl einem völligen Schwunde unterliegen könnte. Diese Vorstellung einer fortschreitenden Degeneration wird jedoch hinfällig, wenn sich herausstellt, dass bei Teleostiern die Spritzlochkieme grossentheils erhalten ist und ihren Kiemencharakter so treu bewabrt, dass sie, wie $\mathrm{H}$. Vir ch ow bemerkt hat, die sperifischen Kiemencharaktere einzelner Familien annimmt (Cit. 8). Hier ist an die beiden $\mathrm{Ar}^{-}$ beiten von Ma r rer zu erinnern (Cit. 4 u. 7), in deren erster die Deckelkieme der Teleostier als Hyoidkieme, in deren zweiter jedoch im Gegensatze dazu sie als Spritzlochkieme angesehen wird. Es lässt sich daher aus dem wollerhaltenen Kiemencharakter kein Schluss gegen die spiraculare Bedeutung entnohmen.

Bezichung zur Spritzlochtasche. Es kommen hier zwei Punkte in Betracht; die rudimentäre Form der Tasche und die Lage der Pseudobranchie zu derselben. Der Umstand, dass die Tasche eine so geringe Entwicklung erführt, mag es plausibel erscheinen lassen, dass auch die Kieme rudimentär bleibt oder vielmehr gar nicht angelegt wird, wie es ja auch in der Darstellung von Balfour und Parker (Cit. 3, S. 429) hervortritt. Wir haben aber eben zu constatiren, dass diese enge Abhängigkeit nicht existirt, vielmehr trot $z$ des Schwundes der Spalte die Kieme bestehen bleiben kann.

Hinsichtlich der Lage bemerkt. Wright (Cit. ō, S. 489), die fragliche Kieme sei in dem von ihm beobachteten Stadium um die ganze Breite des M. adductor hyomandibularis hinter dem Tascheneingang gelegen. Diese Beobachtung wird durch meine 
Erfahrungen sehr wesentlich alogeändert, indem im Stadium V, wo die Anlage in Gestalt eines Wulstes sich bemerkbar macht (Fig. 14), dieser Wulst unterhalb des Einganges in die Tasche, halb vor, halb hinter demselben sich findet. Mit dieser Beobachtung allein ist zwar kein zwingender Beweis, aber doch cin hoher Grad von Wahrscheinlichkeit gegeben, dass es sich um eine Kieme der Spritzlochtasche handle; wir finden, so zu sagen, die Kieme, indem wir sie anf ihrel. Wanderung verfolgen, noch ganz in der Nähe ihres ursprünglichen Platzes, und nur dem Umstande, dass dieselbe so stark verspiitet auftritt, nnd dass der Gang rudimentär ist, haben wir es zwuschreiben, dass wir sie nicht mehr an ihrem richtigen Fleck treffen.

Bezielrung z u den $\mathrm{Ner}$ en: Wir haben es hier mit der Seite des Problems zu thun, welche $R$ a ms a y Wright in die Betrachtung eingeführt hat, und wir haben es diesem Autor zu verdanken, dass auch diese wichtigon Beziehungen zur Sprache gekommen sind; demn in letzter Linic wird es uns nicht genüigen, zu constatiren, ob wir es mit ciner Kieme des 1. oder 2. Visceralbogens zu thun haben, sondern wir werden nur dann cin befriedigendes Bild von den Verschichnugen und Veräindermugen gewimnen, wenn wir die gegenseitigen Verhältnisse aller constituirenden Theile: Skelet, Muskeh, Nerven, Gefäisse, Schleimbant in einem Gesammtbilde vor Augen haben. Das Ergebniss, zu welchem Wi ight durch das Studium der Nerven grelangt, ist, dass die Pseudobranchie ein Stück der Hyoillkieme und nicht die Spritzlochkieme sei.

Wright formulirt seinen Beweis dallin, dass der Glossopharyngeus, der Nerv der ersten Kiemensjalte, nicht nur zu den beiden an diese Spalte grenzenden Halbkiemen in Beriehung trete, sondern auch die Psendobranchic innervire und zwar letztere vermittels seines R. praetrematicus (Cit. 5, S. 490). Von dem $\mathrm{N}$. facialis giebt Wright sodann eine Analyse und Abgrenzung: gegren den Trigeminus, woraus für unsere Zusammenfassung wichtig ist, dass nach der Meinung des genanuten Autors im R. oticus des Nerven der R. praetrematicus des Facialis d. h. der Spritzlochtasche mit enthalten sei und zwar in Gestalt des ventralen zu dem Sinnesepithel des Divertikels (Cit. 5, S. 491) gefundenen Zweiges. Wright bemerkt selbst, dass eine Controle seiner Ansichten durch die Embryologie erwiunscht sei, jedenfalls lat 
Ueber die Entwicklung und morphologische Bedeutung etc. 497

aber die Meinung eines so bewährten-Kenners des Ganoidenkopfes ein grosses Gewicht. Trotz dieser Autorität und trotzdem ich selber über die Verbreitung der Kopfnerven nicht eingehender informirt bin, will es mir doch scheinen, als wenn die Darstellung von Wright sich selber schlïgt, indem er hervorhebt, dass der untere Abschnitt der Hyoidkieme (hyoidean demibranch) nicht vom Glossopharyugeus, sondern vom Facialis innervirt werde (Cit. 5, S. 490 u. 491). Die Erklärung, welche anscheinend mit den Worten gegeben werden soll: "I would attribute this to the interruption of the vascular arch connecting the upper and lower parts of the hyoidean "demibranch" (Cit. 5, S. 149), ist mir nicht recht verständlich, denn innerhalb der Hyoidkieme. (hyoidean demibranch von Wright) giebt es thatsächlich keine Gefüissunterbrechung, weder 'in embryonalen Stadien, wie meine voransgehende Darstellung zeigt, noch im erwachsenen Zustande, wie dié Ausführungen von Johannes M üller lehren und wie ich aus den Injektionspräparaten des Herrn Prof. H. Virchow ersehe, die am frischen Material gemacht sind. Wenn also der H y o idkieme von Lepidosteus ibre Bedentung als Kieme der Vorderwand der ersten Kiemenspalte belassen wird, obwohl der untere Theil derselben rom $\mathrm{F}$ a $\mathrm{cial}$ is innervirt wird, so ist kein zwingender Grund vorhanden, der P s e u d o bra n c hi e den Charakter einer Spritzlochkieme abzusprechen, obwohl sie rom Glossop harynge us versorgt ist. Die logische Nothwendigkeit, dass die Pseudobranchie zum zweiten Bogen gehören muss, scheint mir damit beseitigt und die Möglichkeit hergestellt, dass sie trot\% der Beziehungen zum Glossopharyngeus eine Spritzlochkieme ist. Aufgabe der Untersuchung, sei es der vergleichend anatomischen, sei es der embryologischen, wïrde es nun sein, nachzuweisen, wie diese Verbindung zu Stande gekommen ist. Jedenfalls scheint mir, dass diesen Beziehungen der Kieme zum Nerven nicht eine so entscheidende Bedeutung zukommt, wie Wright ibnen beimisst.

Beziehnungen zu den Gefässen: Johannes Müller hat in seiner bekannten Darstellung (Cit. 2) auf die Verbindung mit den Kopfgefässen die Behauptnng gegründet, dass die Pseudobranchie von Lepidostens der Spritzlochkieme der Selachier entspricht. Die Uebereinstimmung besteht in den ausführenden Gefässen, während die zuführenden Differenzen zeigen. 
Die Aufgabe der ontogenetischen Betrachtung ist die gleiche welche hinsichtlich der 'Teleostier $\mathrm{M}$ a u er und Doh r k klar hingestellt haben, nämlich festzustellen, ob während der Entwicklung dieselben Zustände, welche bei den Selachiren permanent sind, sich auffinden lassen oder, anders ausgedrückt, ob sich erweisen lässt, dass das Gefässstïck, an welchem die Pseudobranchie entsteht, ein Abschnitt des ersten Visceralbogengefässes ist. Um alle einzelnen Züge dieses Problems klar hervortreten zu lassen, müssen zunächst die übercinstimmenden und abweichenden Punkte zwischen Lepidosteus und den Selachiern bervorgeLoben werden.

Die Angelegenheit der ausfübrenden Arterie steht äusserst klar; die A. efferens der Psendobranchie verbindet sich ebenso wie die von Acipenser und den Selachiern mit der Carotis und giebt ausserdem die A. ophthalmica magna (Chorioidalarterie) ab zum Auge. Bei Knochenfischen verhält sich die Sache embryonal anfänglich ebenso, dann aber lüst sich, wie Dohrn darstellt und durch sehr schöne von E d. M c y er gezeichnete Recoustruktionsfignren erläutert bat, die Verbindung mit der Carotis. Lepidosteus, Acipenser, Selachier und frühere embryonale Stadien von Teleostiern verhalten sich also hier übereinstimmend; in einer Beziehung aber bleiben Lepidosteus und Acipenser auf einer primitiveren Stufe stehen, als die Selachier, nämlich darin, dass die mediane Vereinigung der Carotiden, welche hinter der Einmündung der A. efferens spiricularis liegt, bei ihnen ausbleibt.

In den zuführenden Gefässen zeigen die Selachier eine von der Mitte der Basis der Hyoidkieme ausgehende Arterie, welche ihrem Beginne nach als A. efferens lyoidea lateralis und ihrer Endigung nach als $A$. affereus spiracularis bezeichnet werden kann. Die Teleostier besitzen eine ganz andere Gefässversorgung ihrer Pseudobranchie aus einer ventralen Verlängerung der ausfuhrenden Arterie des ersten Bogens. Man hat sich, wie die Arbeiten ron $\mathrm{M}$ a ure $\mathrm{r}$ und $\mathrm{D}$ o h r n zeigen, bemüht, durch Untersuchung embryonaler Stadien von Teleostiern eine Vermittlung. zwischen dem Verhalten dieser Klasse und den Selachiern berzustellen; eine Zwischenform zwischen beiden war jedoch in ebenso überzengender und jedenfalls einfacheren Weise auf vergleichend anatomischem Wege zu gewinnen, nämlich durch Untersuchung der Ganoiden. Bei Acipenser entsteht die A. affer. spir., wie $\mathrm{J}$ o h. 
Ueber die Entwicklung und morphologische Bedeutung etc. 499

M ï ll e r zeigt, aus zwei Zuflüssen, einem aus der ventralen Verlängerung des ersten Kiemenbogengefässes, einem zweiten von der Hyoidkieme. Man kann das damit gegebene Verhalten vielleicht am deutlichsten in der Nomenclatur wiedergeben, wenn man sagt, zwei Gefässe, eine A. branchio-opercularis und A. hyoideoopercularis vereinigen sich zur Bildung der A. afferens spiracularis.

Bei Lepidosteus baben wir nun genau das Gleiche: Ein Gefäss aus der ventralen Verlängerung der ersten Kiemenarterie (A. hyoideo-opercularis von $\mathrm{J} o \mathrm{~h}$. M ti $\mathrm{ll}$ e r) und ein zweites aus der Hyoidkieme vereinigen sich zur Bildung der A. affer. der Psendobranchie, und da die Kieme, welche von diesem Gefäss versorgt wird, bei Acipenser Spritzlochkieme ist, so wird es dadurch wabrscheinlich, dass die Pseudobranchie von Lepidosteus die gleiche Bedeutung hat. Wenn nun auch nach dem Gesagten Lepidosteus ebenso wie Acipenser eine Zwischenstufe zwischen den Selachiern und Teleostiern einzunehmen scheinen, so gebietet doch die Vorsicht, nicht ohne Weiteres die eben erwähnte A. effer. hyoidea derselben, der so charakteristischen A. effer. hyoidea der Selachier gleichzustellen, da sie nicht wie diese in Seitenlinie nach vorn zieht. Hier findet sich nun ein wichtiger Punkt, der auf Grund der Entwicklung zu erörtern ist. Von den im Vorausgehenden (S. 494) zur Sprache gebrachten drei zuftihrenden Arterien $z u$ dem dorsalen Stück des ersten Bogengefässes ist das eine am meisten ventral gelegene die ventrale Verlängerung aus dem ersten Kiemengefäss bezw. deren Fortsetzung, die A. branchio-opercularis; die beiden anderen treten aus dem zweiten Bogengefïss aus, das dorsale früher, das ventrale oder das mittlere der drei Gefässe später; von den beiden zuletzt genannten Gefässen entspricht das untere, später auftretende der definitiven A. hyoideoopercularis, doch hat nach meiner Meinung das obere früher auftretende mehr Anspruch darauf, mit der lateralen A. effer. hyoidea $=$ affer. spir. der Selachier bomologisirt zu werden. Ein strenger Beweis ist jedoch auch dafür nicht vorhanden.

Etwas weiteres, um meine Befunde in ihrer morphologischen Bedeutung zu charakterisiren, weiss ich nicht hinzuzufügen; die Pseudobranchie entsteht thatsächlich an einem Stück des ersten Bogengefässes und erweisst sich damit als Spritzlochkieme. In der A. effer. spir., wie wir nunmehr sagen können, erhält sich ein Stück von dem dorsalen Abschnitt des ersten Bogengefässes, 
aber ateh die $A$. affer. spir. geht aus dem dorsalen Absehnitt des ersten Bogengefässes hervor, welches ron der Stelle an zu rechnen ist, wo das dorsale Randgefäss des Kiemendeckels einmündete (Fig. 12 und 13). Der ventrale Abschnitt verfällt einer capillaren Aufösung, verliert die Verbindung mit dem Trunens und erhält dafür die mit der ventralen Verlängerung aus dem ersten Kiemenbogen.

Somit sind die Verbältnisse mit aller denkbaren Klarheit auf die morphologisch primären Znstände. zurïickgeführt und Scchritt für Schritt abgeleitet und stellen sich mindestens ebenso klar dar, wie bei irgend einer anderen der niederen Wirbelthierklassen. Nirgends hat sich die Nothwendigkeit erwiesen anf unbestimmte "lacunäre Bluträtume" Bezug zal nehmen, wie sic Doh rn (Cit. 6) heranzieht, sondern uiberall handelt es sich von Anfang an nm klare Gefässbahnen, deren Unäinderungen sich mit grösster Wabrscheinlichkeit fiir jede Phase auf ver:̈nderte Bcdingungen nutritiver, respiratorischer und mechanischer Natur zurückführen lassen. Complicationen durch anderweitige Gefüsss. bogen, welche etwa unbekannten hyopothetischen Kiemenbogen zwischen Mandibular- und Hyoidbogen entsprechen könnten, haben sich dabei nicht gefunden, vielmehr sind ntr die Gefüsshogen vorhanden, welche nach dem altbekannten Schema zu erwarten waren. Ebenso wenig hat sich cin primitives Gefäss herallsgestellt, welchem Maurer auf Grund seiner Untersuchungen bei Amphibien (Cit. 7) einen grossen norphologischen Werth locimisst, welches ventral im Hyoidbogen und dorsal in Mandibularbogen seinen Platz hätte, sondern die beiden ersten Bogengefässe sind von der ventralen bis zur dorsalen Seite getrennt vorhanden; nur zeigen sie eine für die späteren Verhältnisse bedentsame Besonderheit darin, dass die Stelle, an welcher sie sich aus einem gemeinsamen Anfangsstück sondern, von Anfang an nach der Seite verschoben ist (Fig. 3).

\section{Erklärung der Abbildungen auf Tafel $\mathbf{X I X}-\mathbf{X X}$.}

Sämmtliche in den Figuren angewandte Bezeichnungen alphabetisch geordnet:

aff. $b r . I=\mathrm{A}$. afferens branchialis $I$.

aff. br. $I I=\mathrm{A}$. afferens branchialis II. aff. $b r . I I I+I V=$ gemeinsamer

Stamm für :

aff. br. $I I I=A$. afferens branchia-

lis III und 
Ueber die Entwicklung und morphologische Bedeutung etc. 501

aff. $b r . I V=$ A. afferens branchialis IV.

aff. $h y .=$ A. afferens hyoidea.

$a f f . s p .=\mathrm{A}$. afferens spiracularis.

an. = Anastomose zwischen are. I und arc. II.

Ao. $=$ Aorta.

arc. $I+I I=$ gemeinsamer Stamm für arc I und are II.

arc. $I=$ Gefäss des 1 . Visceralbogens.

arc. $I I=$ Gefiuss des 2. Visceralbogens.

arc. $I I^{\prime}=$ vorderer Theil des capillaren Anastomosennetzes zw. h. d. u. h.v.

arc. $I I I+I V=$ gemeinsamer Stamm für arc. III u. arc. IV.

arc. $I I I=$ Gefiiss des 1 . Kiemenbogens.

arc. $I V=$ Gefäss des 2 . Kiemenbogens.

arc. $^{\mathbf{1}}=$ einer der Zuflüsse aus dem vom ventralen Randgefitss abgehenden Capiliarnetz. Arc. $I=1$. Kiemenbogen.

Arc. $I I=2$. Kiemenbogen.

$a u .=$ Gehörorgan.

br. $o .=$ A. branchio - opercularis (s. S. 494).

$B u .=$ Bulbus mit den Klappen.

$C a .=$ Carotis.

$c e .=$ Gehirnarterie, A. cerebralis.

$C h .=$ Chorda.

Co. $=$ Herz.

d. $I=$ dorsales Stück des 1. Bogengefässes.

c. $I I=$ dorsal's Stück des 2. Bogengefässes.

eff. br. $I=A$. efferens branchialis $I$.

eff. $b r . I I=$ A. efferens branchialis II. eff. $b r . I I I+I V=$ vereinigter

Stamm von:

eff. $b r . I I I=$ A. efferens branchia-

lis III.

eff. $b r . I V=$ A. efferens branchialis IV.

h. $d .=$ dorsales Randgefäss des

Kiemendecke!s.

$h . d^{\prime} .=$ Zuflüssezum dorsalen Randgefäss (h. d.).

$H e$. = Leber.

$h . v$. - ventrales Randgefäss des Kiemendeckels.

$h . v^{\prime}$. = Seitenzweig des ventralen Randgefässes.

$i . o .=A$. infraorbitalis.

M. $a d d .=$ M. adductor hyomandibularis.

$M d .=$ Mandibula.

$n .=$ A. nasalis

$0 .=$ A. ophthalmica.

$O p .=$ Kiemendeckel.

Per.$=$ Pericardialhöhle.

$P h .=$ Rachenhöhle.

Qu. = Querast des ersten Bogrengefüsses (S. 472).

R. o. = Ramus oticus VII.

$S p .=$ Anlage d. Spritzlochganges.

$S p^{\prime} .=$ Spritzlochtasche.

$S p^{\prime \prime}=$ oberes Ende des Divertikels

des Spritzlochganges.

$T r .=$ Truncus arteriosus.

$T r^{\prime} .=$ Truncus vor dem Abgang des A. effer. br. II.

$V i .=$ Dottersack.

$W .=$ Wulst unterhalb der Anlage des Spritzlochganges.

$V .=$ Venendurchschnitte auf den Querschnittszeichnungen.

$H . k .=$ Hyoidkieme.

$P s_{.}=$Pseudobranchie.

Fig. 1. Querschnitt durch die Anlage des Spritzlochganges einer Larve von $8 \mathrm{~mm}$ (s. S. 466) Vergr. $=40$.

Fig. 2. Vorderende der gleichen Larve aus der Querschnittserie reconstruirt (s. S. 468) Vergr. $=40$. 
Fig. 3. Dasselbe wie Fig. 2 von oben gesehen (s. S. 468). Vergr. $=40$.

Fig. 4. Querschnitt durch die Anlage des Spritzlochganges einer Larve von $10,9 \mathrm{~mm}$ (s. S. 470). Verg'r. $=40$.

Fig. 5. Vorderende der gleichen Larve wie Fig. 4. aus der Querschnittserie reconstruirt (s. S. 471). Vergr. $=40$.

Fig. 6. Dasselbe von oben gesehen. Vercr. 40.

Fig. 7. Querschnitt durch die Anlage des Spritzlochganges einer Larve vom Stadium III. Vergr. $=40$.

Fig. 8. Querschnitt durch die Anlage des Spritzlochganges einer Larve von $16 \mathrm{~mm}$ (s. S. 475 ). Vergr. $=40$.

Fig. 9. Kopf der gleichen Larve wie Fig. 8 aus der Querschnittserie reconstruirt (s. S. 476). Vergr. $=30$.

Fig. 10. Derselbe von oben gesehen. Vergr. $=30$.

Fig. 11. Querschnitt durch den Spritzlochgang eines jungen Thieres von $26 \mathrm{~mm}$ (s. S. 479). Vergr. $=30$.

Fig. 12. Kopf des gleichen Thieres wie in Fig. 11 von der Mitte des Auges bis zum hinteren Rande des Kiemendeckels, von der Seite gesehen; aus der Querschnittserie reconstruirt (s. S. 480). Vergr. $=20$.

Fig. 13. Derselbe von oben gesehen. Vergr. $=20$.

Fig. 14. Seitliche Pharynxwand des gleichen Thieres vom Medianschnitt gesehen nach einer Plattenreconstrulition. Der Kopf ist in derselben Ausdehnung reconstruirt, welche den beiden vorhergehenden Abbildungen entspricht, jedoch ohne Gehirn.

Fig. 15. "Gefässe der Kiemendeckelkieme unč Pseudobranchie" nach Joh. Müller (Cit. 2., Taf V, Fig. 6). Die Bezeichnungen entsprechen denen in den übrigen Figuren.

In den Gefässzeichnungen (Fig. 2, 3, 5, 6, 9, 10, 12, 13) sind die Ebenen der Querschnitte durch Pfeile, die von der Figur abgewandt sind, angedentet. Bei Figur 11 ist die linke Seite des Schnittes berücksichtigt, welche durch die Mitte der pharyngealen Oeffnung des Spritzlochganges geht.

\section{Verzeichniss der in der Arbeit verwertheten und citirten literatur.}

1. Joh. Müller, Vergl. Anatomie der Myxinoiden. Verhandlungen der Berliner Akademie der Wissenschaften. 1839.

2. Derselbe, Ueber den Bau und die Grenzen der Ganoiden und über das natürliche System der Fische. Verhandlungen der Berliner Akademie der Wissenschaften 1846.

3. F. M. Balfour und W. N. Parker, On the structure and deve- 
Ueber die Entwicklung und morphologische Bedeutung etc. 503

lopment of Lepidostens. Phil. Transact. of the R. S. London 1882, vol. 173, p. II.

4. F. Maurer, Fin Beitrag zur Kenntniss der Fseudobranchien der Knochenfische. Morphol. Jahrbuch IX.

5. Ramsay Wright, On the hyomandibular clefts and pseudobranchs of Lepidosteus and Amia. Journ. of Anat. and Phys. 1885.

6. A. Dohrn, Studien zur Urgeschichte des Wirbelthierkörpers. XP. Spritzlochkieme der Selachier, Kiemendeckelkieme der Ganoiden, Pseudobranchie der Teleostier. Mittheilungen aus der zoolog. Station zu Neapel VII. 1886/87.

7. F. Maurer, Die Kiemen und ihre Gefässe bei Anuren und urodelen Amphibien und die Umbildung der beiden ersten Arterienbogen bei Teleostiern. Morph. Jahrbuch XIV.

8. H. Virchow; Ueber die Augengefässe der Selachior und die Verbindung derselben mit den Kopfgefässen. Verhandlungen der Physiolog. Gesellschaft zu Berlin. Jahrgang 1889/90.

9. Derselbe, Embryologische und angiologische Erfahrungen über nordamerikanische Wirbelthiere. Sitzungsberichte der Gesellschaft naturforschender Freunde. Jahrgang 1894.

10. Fr. Fülleborn, Bericht über eine zur Untersuchung der Entwicklung von Amia, Lepidosteus und Necturus unternommene Reise nach Nord-Amerika. Sitzungsberichte der Königl.Preussischen Aliademie der Wissenschaften, Berlin 1894.

\title{
Beobachtungen über die Entwicklungs- geschichte der Nemertinen.
}

\author{
Von
}

\section{Jacob Lebedinsky,}

Privatdocent an der Universität in Odessa.

Hierzu Tafel XXI, XXII, XXII.

Die Nemertinen entwickeln sich, wie bekannt, anf zweierlei Weise. Die einen laufen in der Ontogrenie eine stieng ausgesprochene Metamorphose durch, indem sie die larvalen Formen durchgehen, die anderen entwickeln sich dagegen ohne jede 